\title{
Anticipative calculus with respect to filtered Poisson processes
}

\author{
L. Decreusefond ${ }^{\mathrm{a}, \mathrm{b}, *}$, N. Savy ${ }^{\mathrm{c}}$ \\ a GET, Ecole Nationale Supérieure des Télécommunications, LTCI-UMR 5141, CNRS, 46, rue Barrault, 75634 Paris, France \\ ${ }^{\mathrm{b}}$ ENST - LTCI-UMR 5141 CNRS, 46, rue Barrault, 75634 Paris, France \\ ${ }^{\mathrm{c}}$ Institut de Recherche Mathématiques de Rennes, Université de Rennes 1, 35042 Rennes cedex, France
}

Received 5 March 2004; accepted 22 March 2005

Available online 18 November 2005

\begin{abstract}
We construct the basis of a stochastic calculus for a new class of processes: filtered Poisson processes. These processes are defined by an fBm-like stochastic integral but a Poisson process is subsided to the Brownian motion. We use Malliavin calculus to first construct a gradient then a divergence operator, which will play the role of an anticipative stochastic integral. We study into details the sample-paths regularity of this integral and give an Itô formula for Itô-like processes.
\end{abstract}

(C) 2005 Elsevier SAS. All rights reserved.

\section{Résumé}

Les processus de Poisson filtrés sont définis par une intégrale stochastique d'un noyau déterministe relativement à un processus de Poisson. Ils sont au processus de Poisson ce que le mouvement brownien fractionnaire est au mouvement brownien ordinaire. Nous construisons pour ces processus les bases du calcul anticipatif. Ce travail similaire à celui d'Oksendal et ses collaborateurs sur les processus de sauts de Lévy, emprunte une autre approche que celle du calcul de Hida. Nous introduisons l'opérateur gradient sans utiliser la décomposition en chaos puis nous définissons son adjoint, ce qui nous permet de construire une notion d'intégrale anticipante. Les propriétés de régularité trajectorielle de cette intégrale sont précisées. Nous terminons notre travail par une formule d'Itô pour ces processus.

(c) 2005 Elsevier SAS. All rights reserved.

MSC: 60H05; 60H07; 60G55

Keywords: Anticipative calculus; Malliavin calculus; Poisson process; Shot-noise process; Stochastic integral

Mots-clés : Calcul anticipatif; Calcul de Malliavin ; Processus de Poisson ; Processus shot-noise

\section{Motivation}

Modeling physical phenomena by stochastic processes should satisfy two constraints: a model must capture the prominent features of the phenomenon under consideration but it must also lead to tractable computations. It is thus necessary to always enlarge our catalog of processes for which we can do some calculations. Motivated by potential

\footnotetext{
* Corresponding author.

E-mail addresses: Laurent.Decreusefond@enst.fr (L. Decreusefond), Nicolas.Savy@univ-brest.fr (N. Savy).
} 
applications to mathematical finance, we are interested in stochastic calculus, anticipative or not, with respect to a new class of processes we call filtered Poisson processes. By filtered Poisson process (fpp for short), we mean a process defined by:

$$
N_{t}^{K}=\int_{0}^{t} \int_{\mathbb{R}^{d}} z K(t, s) \omega(\mathrm{d} s, \mathrm{~d} z),
$$

where $\omega$ is a marked Poisson measure, i.e., with a deterministic compensator (or dual predictable projection) $v$ and $K$ a deterministic kernel. When $K$ is of the form $K(t, s)=k(t-s), N^{K}$ is a so-called Shot-Noise process. Such processes provide reasonable models for many phenomena in electronics, hydrology, climatology, telecommunications (see [14,22,23] and references therein), insurance (see [5] and references therein) and finance (see [4] and [21] for a review on this topic).

These processes are intimately related to pure jump Lévy processes. Pure jump Lévy processes have the representation

$$
N_{t}=\int_{0}^{t} \int_{\mathbb{R}} z(\omega(\mathrm{d} s, \mathrm{~d} z)-v(\mathrm{~d} s, \mathrm{~d} z)),
$$

where $v$ is the compensator of $\omega$ and is assumed to be deterministic. Taking $K(t, s)=\mathbf{1}_{[0, t]}(s)$ and $d=1$, a pure jump Lévy process thus appears to be a filtered Poisson process plus a deterministic process. When it comes to stochastic calculus, deterministic processes are in some sense negligible, thus what follows can also be applied to pure jump Lévy processes.

If $K$, in (1), is smooth enough, $N^{K}$ is a semi-martingale so that stochastic calculus with respect to this process can be done inside the classical framework. We want here to construct the tools required to handle the situation of a somehow singular kernel $K$ and the tools required for anticipative calculus. This means that we want to develop a Malliavin calculus for our new class of processes. Such a problem has already been considered in several publications: a first track consists of using chaotic decomposition as in $[13,16]$ and more recently by $[3,8,12]$ for pure jump Lévy processes. Developing and using Hida calculus for pure jump Lévy processes, Di Nunno et al. [8] constructed an anticipative integral and established, among other results, an anticipative Itô formula.

As usual when it comes to Malliavin calculus for jump processes, we can choose another approach and introduce a different gradient operator via a sample-path perturbation (see $[1,16,6])$. That is the way we proceeded here. Still, it must be noted that our definition of the gradient does not coincide with the definition given in [1] or [16]. Actually, our goal is to define a gradient such that its adjoint restricted to predictable processes coincides with the LebesgueStieltjes integral with respect to $\omega-v$. For, we have to "twist" the perturbation in the definition of $D F(h)$, see (2), otherwise we would have additional terms as in [1, Formula 3.11]. The paper is organized as follows. In Section 2, we construct the basis of the Malliavin calculus for marked point processes. The material here is mainly excerpted of [6] though we can establish more properties because of the specific form of the compensator. Once this is done, we develop the stochastic calculus with respect to $N^{K}$ in Section 3. Since $N^{K}$ may have finite variation, we take care to compare Lebesgue-Stieltjes integral with our newly constructed integral. As Theorem 19 shows, they do not coincide. Nevertheless, the divergence is an interesting tool because it is the convenient one to establish the Itô formula by which we conclude this paper. The necessary material about deterministic fractional calculus is recalled in Appendix A.

\section{Stochastic calculus for marked point processes}

\subsection{Introduction}

The space of simple, locally finite on $[0, T] \times \mathbb{R}^{d}(T$ deterministic finite or not $)$ integer-valued measure is denoted by $\Omega$. Assume that we are given a probability measure $\eta$ on $E=\mathbb{R}^{d}$ and a positive, square integrable function, $\lambda$, on $[0, T]$ ( $\lambda$ will both denote the function $s \rightarrow \lambda(s)$ and the measure $\lambda$ of density $\lambda$ with respect to the Lebesgue measure) such that $m:=\inf _{s \in[0, T]} \lambda(s)>0$. We define the probability $\mathbb{P}$ as the unique measure on $\Omega$ such that the 
canonical measure $\omega$ is a Poisson random measure of compensator $v(\mathrm{~d} s, \mathrm{~d} z):=\lambda(s) \mathrm{d} s \eta(\mathrm{d} z)$. The canonical filtration $\mathfrak{F}$ is defined by:

$$
\mathfrak{F}_{0}=\{\emptyset, \Omega\} \quad \text { and } \quad \mathfrak{F}_{t}=\sigma\left\{\int_{0}^{s} \int_{B} \omega(\mathrm{d} s, \mathrm{~d} z), s \leqslant t, B \in \mathcal{B}\left(\mathbb{R}^{d}\right)\right\} .
$$

The predictable $\sigma$-algebra on $\Omega \times R^{+} \times \mathbb{R}^{d}$ is denoted by $\mathcal{P}$. We denote by $T_{n}$ the $n$-th jump time and by $Z_{n}$ the $n$-th mark, this means that

$$
\omega(\mathrm{d} s, \mathrm{~d} z)=\sum_{n \geqslant 1} \delta_{T_{n}, Z_{n}}(s, z) .
$$

The mark point process $N$ is defined by

$$
N_{t}=\sum_{n \in \mathbb{N}^{* *}} Z_{n} \mathbb{I}_{\left[T_{n} \leqslant t\right]}
$$

Following [10], we introduce the following notations: For every measurable and locally bounded or non-negative process $f$,

$$
(f * \mu)_{t}(\omega):=\int_{0}^{t} \int_{\mathbb{R}^{d}} f(s, z)(\omega) \mu(\omega, \mathrm{d} s, \mathrm{~d} z)=\sum_{n \geqslant 1} f\left(T_{n}(\omega), Z_{n}(\omega)\right) \mathbb{I}_{\left[T_{n}(\omega) \leqslant t\right]} .
$$

The process $N$ is of finite variation on every compact of time. Thus, for every measurable and locally bounded or non-negative process $X$ the process defined by:

$$
\left(X^{(S L)} N\right)_{t}(\omega):=\int_{0}^{t} X_{s}(\omega) \mathrm{d} N_{s}(\omega)
$$

exists for a.e. $\omega \in \Omega$ and $t \in \mathbb{R}^{+}$in Stieltjes-Lebesgue way and we have:

$$
\left(X^{(S L)} N\right)_{t}(\omega)=\sum_{n \geqslant 1} Z_{n}(\omega) X_{T_{n}(\omega)}(\omega) \mathbb{I}_{\left[T_{n}(\omega) \leqslant t\right]} .
$$

We recall here the main results of [6].

Definition 1. A functional is said to be cylindric whenever it is of the form

$$
F=f\left(\int_{0}^{T} \int_{\mathbb{R}^{d}} f_{1}(s) g_{1}(z) \omega(\mathrm{d} s, \mathrm{~d} z), \ldots, \int_{0}^{T} \int_{\mathbb{R}^{d}} f_{n}(s) g_{n}(z) \omega(\mathrm{d} s, \mathrm{~d} z)\right),
$$

where $f$ is a bounded twice differentiable function with bounded derivatives, $f_{i} g_{i} \in \mathcal{L}^{2}(v) f_{i}$ is continuously differentiable with bounded derivatives for all $i=1, \ldots, n$. We denote by $\mathcal{S}$ the set of cylindric functionals.

Definition 2. For any functionals $F \in \mathcal{S}$ and any $h \in \mathcal{L}^{2}(v)$, we define $D F(h)$ by

$$
\begin{aligned}
D F(h)= & -\sum_{i=1}^{n} \frac{\partial f}{\partial x_{i}}\left(\int_{0}^{T} \int_{\mathbb{R}^{d}} f_{1}(s) g_{1}(z) \omega(\mathrm{d} s, \mathrm{~d} z), \ldots, \int_{0} \int_{\mathbb{R}^{d}} f_{n}(s) g_{n}(z) \omega(\mathrm{d} s, \mathrm{~d} z)\right) \\
& \times \int_{0}^{T} \int_{\mathbb{R}^{d}} f_{i}^{\prime}(s) g_{i}(z)\left(\frac{1}{\lambda(s)} \int_{0}^{s} h(r, z) \lambda(r) \mathrm{d} r\right) \omega(\mathrm{d} s, \mathrm{~d} z) .
\end{aligned}
$$

The main properties of $D F(h)$ are summarized in the following theorem: 


\section{Theorem 1.}

(1) D is a derivation: for $F, G \in \mathcal{S}$, and $h \in \mathcal{L}^{2}(v)$,

$$
D F G(h)=F D G(h)+G D F(h) .
$$

(2) D satisfies the following integration by parts formula:

$$
\mathbb{E}[D F(h)]=\mathbb{E}\left[F \cdot\left(h^{(S I)}(\omega-v)\right)_{T}\right] .
$$

The particular hypothesis we have here, enables us to simplify the theory developed in [6].

Theorem 2. For all $F \in \mathcal{S}$ there exists a constant $c>0$ such that, for any $h \in \mathcal{L}^{2}(v)$, we have:

$$
\mathbb{E}\left[|D F(h)|^{2}\right] \leqslant c\|h\|_{\mathcal{L}^{2}(v)}^{2} .
$$

In what follows, $c$ denote any irrelevant constant and may vary from line to line.

Proof. Using the boundedness of the functions $f_{i}, f_{i}^{\prime}$ and $g_{i}$ there exists a constant $c$ such that:

$$
\mathbb{E}\left[|D F(h)|^{2}\right] \leqslant c \mathbb{E}\left[\left|\int_{0}^{T} \int_{\mathbb{R}^{d}} \frac{1}{\lambda(s)} \int_{0}^{s} h(r, z) \lambda(r) \mathrm{d} r \omega(\mathrm{d} s, \mathrm{~d} z)\right|^{2}\right] .
$$

Since $\lambda$ is lower-bounded by $m>0$, we have:

$$
\mathbb{E}\left[|D F(h)|^{2}\right] \leqslant c \mathbb{E}\left[\left.\int_{0}^{T} \int_{\mathbb{R}^{d}}^{T}\left[\int_{0}^{s} h(r, z) \lambda(r) \mathrm{d} r\right] \omega(\mathrm{d} s, \mathrm{~d} z)\right|^{2}\right] .
$$

Moreover, using Cauchy-Schwarz inequality,

$$
\begin{aligned}
& \mathbb{E}\left[\left|\int_{0} \int_{\mathbb{R}^{d}} h(s, z) \omega(\mathrm{d} s, \mathrm{~d} z)\right|^{2}\right] \\
& \leqslant 2 \mathbb{E}\left[\left|\int_{0}^{T} h(s, z)(\omega-v)(\mathrm{d} s, \mathrm{~d} z)\right|^{2}\right]+2\left|\iint_{\mathbb{R}^{d}} h(s, z) v(\mathrm{~d} s, \mathrm{~d} z)\right|^{2} \\
& \leqslant 2 \mathbb{E}\left[\iint_{0}^{T} h(s, z)^{2}(\omega-v)(\mathrm{d} s, \mathrm{~d} z)\right] \mathbb{E}\left[\iint_{\mathbb{R}^{d}}^{T}(\omega-v)(\mathrm{d} s, \mathrm{~d} z)\right]+2 \int_{\mathbb{R}^{d}} \int_{\mathbb{R}^{d}} h(s, z)^{2} v(\mathrm{~d} s, \mathrm{~d} z) \\
& \leqslant 2 \mathbb{E}\left[\int_{0}^{T} h(s, z)^{2} v(\mathrm{~d} s, \mathrm{~d} z)\right] v([0, T] \times E)+2 \int_{\mathbb{R}^{d}}^{T} h(s, z)^{2} v(\mathrm{~d} s, \mathrm{~d} z) \\
& \leqslant 2\left(1+v\left([0, T] \times \mathbb{R}^{d}\right)\right) \int_{0}^{T} \int_{\mathbb{R}^{d}} h(s, z)^{2} v(\mathrm{~d} s, \mathrm{~d} z) .
\end{aligned}
$$

We thus get the following relation:

$$
\mathbb{E}\left[|D F(h)|^{2}\right] \leqslant c \int_{0}^{T} \int_{\mathbb{R}^{d}}\left[\int_{0}^{s} h(r, z) \lambda(r) \mathrm{d} r\right]^{2} v(\mathrm{~d} s, \mathrm{~d} z) .
$$


Using the special form of the measure $v$, we can write:

$$
\begin{aligned}
\iint_{\mathbb{R}^{d}}^{T}\left[\int_{0}^{s} h(r, z) \lambda(r) \mathrm{d} r\right]^{2} \lambda(s) \eta(\mathrm{d} z) \mathrm{d} s & \leqslant \int_{0}^{T} \int_{\mathbb{R}^{d}}\left(\int_{0}^{s} h^{2}(r, z) \lambda(r) \mathrm{d} r\right)\left(\int_{0}^{s} \lambda^{2}(r) \mathrm{d} r\right) \lambda(s) \eta(\mathrm{d} z) \mathrm{d} s \\
& \leqslant \iint_{0}^{T}\left(\int_{\mathbb{R}^{d}}^{T} h^{2}(r, z) \lambda(r) \mathrm{d} r\right)\left(\int_{0}^{T} \lambda^{2}(r) \mathrm{d} r\right) \lambda(s) \eta(\mathrm{d} z) \mathrm{d} s \\
& \leqslant\|\lambda\|_{2}^{2}\|\lambda\|_{1} \iint_{0}^{T} h_{\mathbb{R}^{d}}^{2}(r, z) \lambda(r) \mathrm{d} r \eta(\mathrm{d} z) .
\end{aligned}
$$

From that and from relation (4), we infer that

$$
\mathbb{E}\left[|D F(h)|^{2}\right] \leqslant c \int_{0}^{T} \int_{\mathbb{R}^{d}} h^{2}(r, z) \lambda(r) \mathrm{d} r \eta(\mathrm{d} z) .
$$

The proof is thus complete.

Theorem 3. For any $F \in \mathcal{S}$, there exists $\nabla F \in \mathbb{L}^{2}([0, T] \times E \times \Omega, v \otimes d \mathbb{P})$ measurable with respect to the three variables such that:

$$
D F(h)=\langle\nabla F, h\rangle_{\mathcal{L}^{2}(v)} \quad \text { for all } h \in \mathcal{L}^{2}(v)
$$

Proof. Let $F \in \mathcal{S}$ be fixed. Consider the application:

$$
\begin{aligned}
\phi_{F}: \mathcal{L}^{2}(v) \otimes \mathbb{L}^{2}(\Omega) & \rightarrow \mathbb{R}, \\
h \otimes G & \rightarrow \mathbb{E}[G D F(h)] .
\end{aligned}
$$

It is clearly linear, let us show its continuity. One considers the projective tensor product (see Köthe [11] Section 41 for details) equipped with the following semi-norm:

$$
\|z\|=\inf \sum_{i=1}^{n}\left\|h_{i}\right\|_{\mathcal{L}^{2}(v)}\left\|G_{i}\right\|_{2}
$$

where the 'inf' is taken over all the possible decompositions of $z$ in the form $z=\sum_{i=1}^{n} h_{i} \otimes G_{i}$. It is then sufficient to show the existence of a constant $c$ such that:

$$
\left|\phi_{F}\left(\sum_{i=1}^{n} h_{i} \otimes G_{i}\right)\right| \leqslant c\left\|\sum_{i=1}^{n} h_{i} \otimes G_{i}\right\| .
$$

According to Hölder inequality and to the previous theorem, we have

$$
\begin{aligned}
\left|\phi_{F}\left(\sum_{i=1}^{n} h_{i} \otimes G_{i}\right)\right| & \leqslant \sum_{i=1}^{n}\left|\mathbb{E}\left[G_{i} D F\left(h_{i}\right)\right]\right| \leqslant \sum_{i=1}^{n}\left\|G_{i}\right\|_{2}\left\|D F\left(h_{i}\right)\right\|_{2} \\
& \leqslant c \sum_{i=1}^{n}\left\|G_{i}\right\|_{2}\left\|h_{i}\right\|_{\mathcal{L}^{2}(v)} \leqslant c\left\|\sum_{i=1}^{n} h_{i} \otimes G_{i}\right\| .
\end{aligned}
$$

Thus, $\phi_{F}$ is a continuous linear form. Using the identification of tensor product of $\mathcal{L}^{2}$ type spaces:

$$
\mathbb{L}^{2}\left(\Omega \times[0, T] \times \mathbb{R}^{d} ; \mathrm{d} \mathbb{P} \otimes v\right) \sim \mathcal{L}^{2}(v) \otimes \mathbb{L}^{2}(\Omega),
$$

we can invoke the Riesz representation theorem which ensures the existence of a measurable random variable $\nabla F \in$ $\mathbb{L}^{2}\left([0, T] \times \mathbb{R}^{d} \times \Omega, v \otimes \mathrm{d} \mathbb{P}\right)$ such that: 


$$
\begin{aligned}
\phi_{F}(G \otimes h) & =\langle\nabla F ; G \otimes h\rangle_{\mathbb{L}^{2}(\Omega) \otimes \mathcal{L}^{2}(v)} \\
& =\mathbb{E}\left[G\langle\nabla F ; h\rangle_{\mathcal{L}^{2}(v)}\right]=\mathbb{E}[G D F(h)] .
\end{aligned}
$$

The proof is thus complete.

Theorem 4. The application $F \rightarrow \nabla F$ is closable in $\mathbb{L}^{2}(\mathbb{P})$.

Proof. Let $\left\{F_{n}, n \geqslant 1\right\}$ a sequence of $\mathcal{S}$ such that $F_{n}$ tends to 0 in $\mathbb{L}^{2}(\mathbb{P})$ and such that $\nabla F_{n}$ tends to a limit called $L$. For any $G \in \mathcal{S}$ and any $h \in \mathcal{L}^{2}(v)$ we have:

$$
\begin{aligned}
\mathbb{E}[\langle L, h\rangle \cdot G] & =\lim _{n \rightarrow \infty} \mathbb{E}\left[\left\langle\nabla F_{n}, h\right\rangle_{\mathcal{L}^{2}(v)} \cdot G\right] \\
& =\lim _{n \rightarrow \infty} \mathbb{E}\left[\left\langle\nabla F_{n} \cdot G, h\right\rangle_{\mathcal{L}^{2}(v)}\right] \\
& =\lim _{n \rightarrow \infty}\left(\mathbb{E}\left[\left\langle\nabla\left(F_{n} G\right), h\right\rangle_{\mathcal{L}^{2}(v)}\right]-\mathbb{E}\left[\left\langle\nabla G \cdot F_{n}, h\right\rangle_{\mathcal{L}^{2}(v)}\right]\right) \\
& =\lim _{n \rightarrow \infty}\left(\mathbb{E}\left[\left\langle\nabla\left(F_{n} G\right), h\right\rangle_{\mathcal{L}^{2}(v)}\right]-\mathbb{E}\left[\langle\nabla G, h\rangle_{\mathcal{L}^{2}(v)} F_{n}\right]\right) \\
& =\lim _{n \rightarrow \infty}\left(\mathbb{E}\left[F_{n} G \cdot\left(h^{*}(\omega)(\omega)\right)_{T}\right]-\mathbb{E}\left[\langle\nabla G, h\rangle_{\mathcal{L}^{2}(v)} F_{n}\right]\right) \\
& =0,
\end{aligned}
$$

since $F_{n}$ tends to 0 in $L^{2}(\mathbb{P})$. As $\mathcal{S}$ is dense in $\mathbb{L}^{2}(\mathbb{P})$, we infer that $L=0 \mathbb{P}$-a.e.

Definition 3. On $\mathcal{S}$, we introduce the norm: for any $F \in \mathcal{S}$,

$$
\|F\|_{2,1}^{2}=\|F\|_{2}^{2}+\mathbb{E}\left[\|\nabla F\|_{\mathcal{L}^{2}(v)}^{2}\right]
$$

and the space $\mathbb{D}_{2,1}$, the closure of $\mathcal{S}$ with respect to this norm.

The usual properties then follow (see [6]): for $(F, G) \in\left(\mathbb{D}_{2,1}\right)^{2}$, and $\phi \in \mathcal{C}_{b}^{1}$, we have:

$$
\nabla F G=G \nabla F+F \nabla G \text { and } \nabla(\phi(F))=\phi^{\prime}(F) \nabla F .
$$

Definition 4. Let $\zeta$ be an $\mathcal{L}^{2}(v)$-valued random variable, it is in the domain of $\delta(\operatorname{dom}(\delta))$ iff, there exists $c$ such that for any $F \in \mathcal{S}$ we have:

$$
|\mathbb{E}[D F(\zeta)]| \leqslant c\|F\|_{2} .
$$

In this case, $\delta(\zeta)$ is defined by:

$$
\mathbb{E}[F \delta(\zeta)]=\mathbb{E}[D F(\zeta)]
$$

Proposition 1. Any element of $\mathcal{L}^{2}(v)$ belongs to $\operatorname{dom}(\delta)$ and $\delta(h)=\left(h^{*}(\omega-v)\right)_{T}$.

Proof. Since, for $h \in \mathcal{L}^{2}(v)$, we have:

$$
\mathbb{E}[D F(h)]=\mathbb{E}\left[F \cdot\left(h^{(S I)}(\omega-v)\right)_{T}\right],
$$

it is clear that $\mathcal{L}^{2}(v) \subset \operatorname{dom}(\delta)$ and by identification that $\delta(h)=\left(h^{*}(\omega-v)\right)_{T}$.

Theorem 5. For any $a \in \mathcal{S}$ and any $h \in \mathcal{L}^{2}(v), a \cdot h$ belongs to $\operatorname{dom}(\delta)$ and

$$
\delta(a h)=a \delta(h)-D a(h) .
$$

Proof. For $F \in \mathcal{S}$, it is easy to see, by definition of $D F(h)$, that if $a$ is a random variable, we have:

$$
D F(a h)=a D F(h) \text {. }
$$


Moreover, since $\mathcal{S}$ is an algebra, we get:

$$
\begin{aligned}
\mathbb{E}[D F(a h)] & =\mathbb{E}[a D F(h)]=\mathbb{E}[D(a F)(h)-F D(a)(h)] \\
& =\mathbb{E}[F a \delta(h)]-\mathbb{E}[F D(a)(h)] .
\end{aligned}
$$

It is then clear that:

$$
|\mathbb{E}[D F(a \zeta)]| \leqslant c\|F\|_{2},
$$

this shows that $a \cdot h \in \operatorname{dom}(\delta)$ and that $\delta(a h)=a \delta(h)-D a(h)$.

Now, we generalize the previous construction by considering $\mathcal{L}^{2}(v)$-valued random variable. The set of cylindrical processes $\mathcal{S}\left(\mathcal{L}^{2}(v)\right)$ is the set of processes of the form:

$$
\Phi=\sum_{i=1}^{k} F_{i} v_{i}, \quad F_{i} \in \mathcal{S}, v_{i} \in \mathcal{L}^{2}(v) .
$$

By definition,

$$
D \Phi(h)=\sum_{i=1}^{k} D F_{i}(h) \otimes v_{i}, \quad F_{i} \in \mathcal{S}, v_{i} \in \mathcal{L}^{2}(v) .
$$

Proposition 2. For any $\Phi \in \mathcal{S}\left(\mathcal{L}^{2}(v)\right), \nabla \Phi$ is an Hilbert-Schmidt operator.

Proof. Note first that the map $f \mapsto \int_{0} f(r) \lambda(r) \mathrm{d} r$ is Hilbert-Schmidt from $\mathcal{L}^{2}(d s)$ into itself. Indeed, this can be written

$$
\int_{0}^{T} \mathbf{1}_{[0, t]}(r) \lambda(r) f(r) \mathrm{d} r
$$

and the kernel $(t, r) \mapsto \mathbf{1}_{[0, t]}(r) \lambda(r)$ is clearly square integrable on $[0,1]^{2}$.

Let $\left(a_{j}, j \geqslant 1\right)$ and $\left(b_{j}, j \geqslant 1\right)$ be CONB of respectively $\mathcal{L}^{2}(\lambda)$ and $\mathcal{L}^{2}(\eta)$. It is clear that

$$
\begin{aligned}
\mathbb{E}\left[\sum_{j, k=1}^{\infty}\left\langle D \Phi\left(a_{j} \otimes b_{k}\right) ; D \Phi\left(a_{j} \otimes b_{k}\right)\right\rangle_{\mathcal{L}^{2}(v)}\right] & \leqslant c \mathbb{E}\left[\sum_{j, k=1}^{\infty}\left[\int_{0}^{T} \frac{1}{\lambda(s)} \int_{0}^{s} a_{j}(r) b_{k}(z) \lambda(r) \mathrm{d} r \omega(\mathrm{d} s, \mathrm{~d} z)\right]^{2}\right] \\
& \leqslant c \mathbb{E}\left[\sum_{j=1}^{\infty} \iint_{0}^{T}\left(\int_{0}^{s} a_{j}(r) \lambda(r) \mathrm{d} r\right)^{2} \mathrm{~d} s\right],
\end{aligned}
$$

and the result follows.

To motivate the following, recall that in the Brownian setting, it is well known that

$$
\mathbb{E}\left[\delta(u)^{2}\right]=\mathbb{E}\left[\|u\|^{2}\right]+\mathbb{E}[\operatorname{trace}(\nabla u \circ \nabla u)] .
$$

Unfortunately, this property does not hold any longer in our situation. We want to find its analog. On one hand, one can keep the analog of the second term of (8) but we have to introduce an operator $\Gamma$ such that:

$$
\mathbb{E}\left[\delta(u)^{2}\right]=\mathbb{E}\left[\langle u, \Gamma u\rangle_{\mathcal{L}^{2}(v)}\right]+\mathbb{E}[\operatorname{trace}(\nabla u \circ \nabla u)] .
$$

This is the main result of Theorem 6. On the other hand, one can keep the analog of the first term of (8) but we have to define another gradient $\hat{\nabla}$ such that:

$$
\mathbb{E}\left[\delta(u)^{2}\right]=\mathbb{E}\left[\|u\|_{\mathcal{L}^{2}(\lambda)}^{2}\right]+\mathbb{E}[\operatorname{trace}(\hat{\nabla} u \circ \hat{\nabla} u)] .
$$


This is the result of Theorem 10, which is valid only when one does not have marks. The gradient $\hat{\nabla}$ is easily linked with the covariant derivative introduced by Privault in [15,17-19].

We need to introduce a few notations. Consider the following space $\mathcal{H}^{\nu}$ :

$$
\mathcal{H}^{v}=\left\{h \in \mathcal{L}^{2}(v): \frac{\partial h}{\partial s} \in \mathcal{L}^{2}(v)\right\}
$$

equipped with the following inner product:

$$
\langle g, h\rangle_{\mathcal{H}^{v}}=\langle g, h\rangle_{\mathcal{L}^{2}(v)}+\left\langle\frac{\partial g}{\partial s}, \frac{\partial h}{\partial s}\right\rangle_{\mathcal{L}^{2}(v)} .
$$

Consider $\left\{\varepsilon_{i}: i \in \mathbb{N}^{*}\right\}$ a CONB of $\mathcal{H}^{\nu}$. Obviously $\mathcal{H}^{\nu} \subset \mathcal{L}^{2}(v)$ and define with obvious notations $\mathcal{S}\left(\mathcal{H}^{\nu}\right)$.

Proposition 3. Let us consider the following operator:

$$
\begin{aligned}
\Gamma: \mathcal{S}\left(\mathcal{H}^{\nu}\right) & \rightarrow \mathbb{L}^{2}(\Omega \times[0, T] \times E, \mathrm{~d} \mathbb{P} \otimes \nu), \\
\zeta & \rightarrow \sum_{i=1}^{\infty}\left\langle\zeta, \varepsilon_{i}\right\rangle_{\mathcal{H}^{\nu}} \Gamma\left(\varepsilon_{i}\right)
\end{aligned}
$$

with $\Gamma\left(\varepsilon_{i}\right)=\nabla\left(\delta\left(\varepsilon_{i}\right)\right)$. Then, $\Gamma$ is continuous.

Proof. Let us show that for any $h \in \mathcal{S}\left(\mathcal{H}^{v}\right)$,

$$
\mathbb{E}\left[\|\nabla(\delta(h))\|_{\mathcal{L}^{2}(v)}^{2}\right]<\infty .
$$

First of all, let $h \in \mathcal{H}^{\nu}$, we have:

$$
\|\nabla(\delta(h))\|_{\mathcal{L}^{2}(v)}^{2}=\sum_{i=1}^{\infty}\left\langle\nabla(\delta(h)),\left.e_{i}\right|_{\mathcal{L}^{2}(v)} ^{2}=\sum_{i=1}^{\infty} D(\delta(h))\left(e_{i}\right)^{2} .\right.
$$

But $\delta(h)$ is cylindrical, so we can write:

$$
\begin{aligned}
\mathbb{E}\left[\|\nabla(\delta(h))\|_{\mathcal{L}^{2}(v)}^{2}\right] & \leqslant \sum_{i=1}^{\infty} \mathbb{E}\left[\left[\int_{0}^{T} \frac{\partial h}{\partial s}(s, z) \frac{1}{\lambda(s)} \int_{0}^{s} e_{i}(r, z) \lambda(r) \mathrm{d} r \omega(\mathrm{d} s, \mathrm{~d} z)\right]^{2}\right] \\
& \leqslant \sum_{i=1}^{\infty} \iint_{0}^{T}\left[\frac{\partial h}{\partial s}(s, z) \frac{1}{\lambda(s)} \int_{0}^{s} e_{i}(r, z) \lambda(r) \mathrm{d} r\right]^{2} v(\mathrm{~d} s, \mathrm{~d} z) \\
& \leqslant \int_{0}^{T}\left[\frac{\partial h^{d}}{\partial s}(s, z) \frac{1}{\lambda(s)^{2}} \sum_{i=1}^{\infty}\left\langle e_{i}, \mathbb{I}_{[0, T]}\right\rangle_{\mathcal{L}^{2}(v)}^{2}\right] v(\mathrm{~d} s, \mathrm{~d} z) \\
& \leqslant \frac{1}{m^{2}} \int_{0}^{T} \int_{\mathbb{R}^{d}} \frac{\partial h^{2}}{\partial s}(s, z)\left\|\mathbb{I}_{[0, T]}\right\|_{\mathcal{L}^{2}(v)}^{2} v(\mathrm{~d} s, \mathrm{~d} z) \leqslant \frac{1}{m^{2}} \int_{0}^{T} \int_{\mathbb{R}^{d}} \frac{\partial h^{2}}{\partial s}(s, z) v(\mathrm{~d} s, \mathrm{~d} z) \\
& \leqslant \frac{1}{m^{2}}\left\|\frac{\partial h}{\partial s}\right\|_{\mathcal{L}^{2}(v)}^{2} .
\end{aligned}
$$

We thus have:

$$
\|\delta(h)\|_{2,1}=\mathbb{E}\left[\delta(h)^{2}\right]+\mathbb{E}\left[\|\nabla(\delta(h))\|_{\mathcal{L}^{2}(v)}^{2}\right] \leqslant\|h\|_{\mathcal{L}^{2}(v)}^{2}+\frac{1}{m^{2}}\left\|\frac{\partial h}{\partial s}\right\|_{\mathcal{L}^{2}(v)}^{2} \leqslant\left(1 \wedge \frac{1}{m^{2}}\right)\|h\|_{\mathcal{H}^{v}}^{2} .
$$


$\delta: \mathcal{H}^{\nu} \rightarrow \mathbb{D}_{2,1}$ is thus continuous and $\nabla: \mathbb{D}_{2,1} \rightarrow \mathbb{L}^{2}(\Omega \times[0, T] \times E, \mathrm{~d} \mathbb{P} \otimes v)$ is also continuous by construction. Then,

$$
\nabla \circ \delta: \mathcal{H}^{v} \rightarrow \mathbb{L}^{2}(\Omega \times[0, T] \times E, \mathrm{~d} \mathbb{P} \otimes v)
$$

is a linear continuous application, i.e., there exists a constant $c$ such that, for any $h \in \mathcal{H}^{\nu}$ :

$$
\mathbb{E}\left[\|\nabla(\delta(h))\|_{\mathcal{L}^{2}(v)}^{2}\right] \leqslant c\|h\|_{\mathcal{H}^{v}}^{2}
$$

and thus for any $i \in \mathbb{N}^{*}$

$$
\mathbb{E}\left[\left\|\nabla\left(\delta\left(\varepsilon_{i}\right)\right)\right\|_{\mathcal{L}^{2}(v)}^{2}\right] \leqslant c .
$$

Consider now $\zeta \in \mathcal{S}\left(\mathcal{H}^{\nu}\right), \zeta=F h$ with $F \in \mathcal{S}$ (thus bounded) and $h \in \mathcal{H}^{\nu}$. Then, we have:

$$
\begin{aligned}
\mathbb{E}\left[\|\Gamma(\zeta)\|_{\mathcal{L}^{2}(v)}^{2}\right] & =\mathbb{E}\left[\left\|\sum_{i=1}^{\infty}\left\langle\zeta, \varepsilon_{i}\right\rangle_{\mathcal{H}^{v}} \Gamma\left(\varepsilon_{i}\right)\right\| \|_{\mathcal{L}^{2}(v)}\right]^{2} \leqslant\|F\|_{\infty}^{2} \sum_{i=1}^{\infty}\left\langle h, \varepsilon_{i}\right\rangle_{\mathcal{H}^{v}}^{2} \mathbb{E}\left[\left\|\Gamma\left(\varepsilon_{i}\right)\right\|_{\mathcal{L}^{2}(v)}\right]^{2} \\
& \leqslant c\|F\|_{\infty}^{2} \sum_{i=1}^{\infty}\left\langle h, \varepsilon_{i}\right\rangle_{\mathcal{H}^{v}}^{2} \leqslant c\|F\|_{\infty}^{2}\|h\|_{\mathcal{H}^{v}}^{2}<\infty .
\end{aligned}
$$

The proof is thus complete.

\section{Theorem 6.}

(1) For any $\zeta \in \mathcal{S}\left(\mathcal{H}^{v}\right)$ we have:

$$
\delta(\zeta)=\sum_{i=1}^{\infty}\left[\left\langle\zeta, \varepsilon_{i}\right\rangle_{\mathcal{H}^{v}} \delta\left(\varepsilon_{i}\right)-\left\langle\left\langle\nabla . \zeta, \varepsilon_{i}\right\rangle_{\mathcal{H}^{v}}, \varepsilon_{i}(\cdot)\right\rangle_{\mathcal{L}^{2}(v)}\right] .
$$

(2) For $\zeta \in \mathcal{S}\left(\mathcal{H}^{\nu}\right)$ we have:

$$
\nabla_{*} \delta(\zeta)=\sum_{i=1}^{\infty}\left\langle\zeta, \varepsilon_{i}\right\rangle_{\mathcal{H}^{\nu}} \nabla_{*}\left(\delta\left(\varepsilon_{i}\right)\right)+\delta\left(\nabla_{*} \zeta\right)
$$

* indicates the free variable.

(3) For any $\zeta \in S\left(\mathcal{H}^{v}\right)$ we have:

$$
\mathbb{E}\left[\delta(\zeta)^{2}\right]=\mathbb{E}\left[\langle\zeta ; \Gamma \zeta\rangle_{\mathcal{L}^{2}(v)}\right]+\mathbb{E}[\operatorname{trace}(\nabla \zeta \circ \nabla \zeta)]
$$

Proof. To prove the first point, we reproduce the proof of Theorem 5. Furthermore, by linearity and continuity of $\delta$ :

$$
\delta(\zeta)=\sum_{i=1}^{\infty} \delta\left(\left\langle\zeta ; \varepsilon_{i}\right\rangle_{\mathcal{H}^{v}} \varepsilon_{i}\right)
$$

Applying the first point of this theorem with $a=\left\langle\zeta ; \varepsilon_{i}\right\rangle_{\mathcal{H}^{v}}$ we get:

$$
\delta(\zeta)=\sum_{i=1}^{\infty}\left[\left\langle\zeta ; \varepsilon_{i}\right\rangle \mathcal{H}^{\nu} \delta\left(\varepsilon_{i}\right)-\left\langle\nabla .\left(\left\langle\zeta ; \varepsilon_{i}\right\rangle_{\mathcal{H}^{v}}\right), \varepsilon_{i}(\cdot)\right\rangle_{\mathcal{L}^{2}(v)}\right] .
$$

Since $\varepsilon_{i}$ is deterministic, we can invert the inner product and the gradient and write:

$$
\left\langle\nabla .\left(\left\langle\zeta ; \varepsilon_{i}\right\rangle \mathcal{H}^{v}\right), \varepsilon_{i}(\cdot)\right\rangle_{\mathcal{L}^{2}(v)}=\left\langle\left\langle\nabla . \zeta, \varepsilon_{i}\right\rangle_{\mathcal{H}^{v}}, \varepsilon_{i}(\cdot)\right\rangle_{\mathcal{L}^{2}(v)},
$$

so that the result holds.

On the one hand we know that:

$$
\delta(\zeta)=\sum_{i=1}^{\infty}\left[\left\langle\zeta, \varepsilon_{i}\right\rangle_{\mathcal{H}^{v}} \delta\left(\varepsilon_{i}\right)-\left\langle\left\langle\nabla . \zeta, \varepsilon_{i}\right\rangle_{\mathcal{H}^{v}}, \varepsilon_{i}(\cdot)\right\rangle_{\mathcal{L}^{2}(v)}\right]
$$


On the other hand:

$$
\delta\left(\nabla_{*} \zeta\right)=\sum_{i=1}^{\infty}\left[\left\langle\nabla_{*} \zeta, \varepsilon_{i}\right\rangle_{\mathcal{H}^{\nu}} \delta\left(\varepsilon_{i}\right)-\left\langle\left\langle\nabla .\left(\nabla_{*}\right) \zeta, \varepsilon_{i}\right\rangle_{\mathcal{H}^{\nu}}, \varepsilon_{i}(\cdot)\right\rangle_{\mathcal{L}^{2}(v)}\right] .
$$

Applying $\nabla$ to both sides of (13), we get:

$$
\begin{aligned}
\nabla_{*}(\delta(\zeta)) & =\sum_{i=1}^{\infty}\left[\left\langle\zeta, \varepsilon_{i}\right\rangle_{\mathcal{H}^{\nu}} \nabla_{*}\left(\delta\left(\varepsilon_{i}\right)\right)+\nabla_{*}\left(\left\langle\zeta, \varepsilon_{i}\right\rangle_{\mathcal{H}^{v}}\right) \delta\left(\varepsilon_{i}\right)-\nabla_{*}\left\langle\left\langle\nabla \zeta, \varepsilon_{i}\right\rangle_{\mathcal{H}^{v}}, \varepsilon_{i}\right\rangle_{\mathcal{L}^{2}(v)}\right] \\
& =\sum_{i=1}^{\infty}\left[\left\langle\nabla_{*} \zeta, \varepsilon_{i}\right\rangle_{\mathcal{H}^{\nu}} \delta\left(\varepsilon_{i}\right)-\left\langle\nabla_{*}\left\langle\nabla \zeta, \varepsilon_{i}\right\rangle_{\mathcal{H}^{v}}, \varepsilon_{i}(\cdot)\right\rangle_{\mathcal{L}^{2}(v)}\right]+\sum_{i=1}^{\infty}\left\langle\zeta, \varepsilon_{i}\right\rangle_{\mathcal{H}^{v}} \nabla_{*}\left(\delta\left(\varepsilon_{i}\right)\right) \\
& =\sum_{i=1}^{\infty}\left[\left\langle\nabla_{*} \zeta, \varepsilon_{i}\right\rangle_{\mathcal{H}^{\nu}} \delta\left(\varepsilon_{i}\right)-\left\langle\left\langle\nabla_{*} \nabla \zeta, \varepsilon_{i}\right\rangle_{\mathcal{H}^{v}}, \varepsilon_{i}(\cdot)\right\rangle_{\mathcal{L}^{2}(v)}\right]+\sum_{i=1}^{\infty}\left\langle\zeta, \varepsilon_{i}\right\rangle_{\mathcal{H}^{v}} \nabla_{*}\left(\delta\left(\varepsilon_{i}\right)\right)
\end{aligned}
$$

According to (14), we get the second formula.

We are now in position to compute the second moment of $\delta(\zeta)$. According to the previous results, we have:

$$
\begin{aligned}
\mathbb{E}\left[\delta(\zeta)^{2}\right] & =\mathbb{E}[\delta(\zeta) \delta(\zeta)]=\mathbb{E}\left[\left\langle\zeta_{*}, \nabla_{*} \delta(\zeta)\right\rangle_{\mathcal{L}^{2}(v)}\right] \\
& =\mathbb{E}\left[\left\langle\zeta_{*}, \sum_{i=1}^{\infty}\left\langle\zeta, \varepsilon_{i}\right\rangle_{\mathcal{H}^{v}} \nabla_{*}\left(\delta\left(\varepsilon_{i}\right)\right)\right\rangle_{\mathcal{L}^{2}(v)}\right]+\mathbb{E}\left[\left\langle\zeta_{*}, \delta\left(\nabla_{*}(\zeta)\right)\right\rangle_{\mathcal{L}^{2}(v)}\right] \\
& =\mathbb{E}\left[\left\langle\zeta_{*}, \Gamma \zeta\right\rangle_{\mathcal{L}^{2}(v)}\right]+\mathbb{E}\left[\left\langle\zeta_{*}, \delta\left(\nabla_{*}(\zeta)\right)\right\rangle_{\mathcal{L}^{2}(v)}\right]
\end{aligned}
$$

Furthermore,

$$
\begin{aligned}
\mathbb{E}\left[\left\langle\zeta_{*}, \delta\left(\nabla_{*}(\zeta)\right)\right\rangle_{\mathcal{L}^{2}(v)}\right] & =\mathbb{E}\left[\iint_{0}^{T} \zeta_{\mathbb{R}^{d}} \delta\left(\nabla_{s, z} \zeta\right) v(\mathrm{~d} s, \mathrm{~d} z)\right]=\mathbb{E}\left[\iint_{0}^{T} \int_{\mathbb{R}^{d}}^{T} \int_{\mathbb{R}^{d}} \nabla_{u, v} \zeta_{s, z} \nabla_{s, z} \zeta_{u, v} v(\mathrm{~d} u, \mathrm{~d} v) v(\mathrm{~d} s, \mathrm{~d} z)\right] \\
& =\mathbb{E}[\operatorname{trace}(\nabla \zeta \circ \nabla \zeta)],
\end{aligned}
$$

the proof is thus complete.

Consider, for $\Phi \in \mathcal{S}\left(\mathcal{H}^{\nu}\right)$ the following norm:

$$
\left[\|\Phi\|_{2,1}^{\Gamma}\right]^{2}=\frac{1}{2}\left(\mathbb{E}\left[\|\Phi\|_{\mathcal{L}^{2}(v)}^{2}\right]+\mathbb{E}\left[\|\Gamma \Phi\|_{\mathcal{L}^{2}(v)}^{2}\right]\right)+\mathbb{E}\left[\|\nabla \Phi\|_{\mathcal{L}^{2}(v) \otimes \mathcal{L}^{2}(v)}^{2}\right]
$$

Consider $\mathbb{D}_{2,1}^{\Gamma}\left(\mathcal{H}^{v}\right)$ the closure of $\mathcal{S}\left(\mathcal{H}^{v}\right)$ with respect to this norm.

Remark 1. In the Brownian framework, $\Gamma$ is equal to identity and we get the usual norm $\mathbb{D}_{2,1}$.

Proposition 4. We have:

$$
\mathbb{D}_{2,1}^{\Gamma}\left(\mathcal{H}^{v}\right) \subset \operatorname{dom}(\delta) .
$$

Proof. Consider $\zeta \in \mathbb{D}_{2,1}^{\Gamma}\left(\mathcal{H}^{\nu}\right)$ and $\left\{\zeta_{n}: n \in \mathbb{N}^{*}\right\}$ a sequence of $\mathcal{S}\left(\mathcal{H}^{\nu}\right)$ which tends to $\zeta$ in $\mathbb{D}_{2,1}\left(\mathcal{H}^{v}\right)$. For any $F \in \mathcal{S}$, we have:

$$
\left|\mathbb{E}\left[D F\left(\zeta_{n}\right)\right]\right|=\left|\mathbb{E}\left[F \delta\left(\zeta_{n}\right)\right]\right| \leqslant\left\|\delta\left(\zeta_{n}\right)\right\|_{2}\|F\|_{2} \leqslant\left\|\zeta_{n}\right\|_{2,1}^{\Gamma}\|F\|_{2}
$$

according to (12) and the result follows.

We now define the integral with respect to $\tilde{N}$. 
Definition 5. For any $u$ from $[0,1]$ into $\mathbb{R}$, we set $\tilde{u}(s, z)=z u(s)$. If $\tilde{u} \in \operatorname{dom}(\delta)$, we define $\delta^{\widetilde{N}}(u)$ by

$$
\delta^{\widetilde{N}}(u)=\delta(\tilde{u}),
$$

and $\tilde{\lambda}$ is denoting the measure:

$$
\tilde{\lambda}(\mathrm{d} s)=\int_{\mathbb{R}^{d}} z^{2} v(\mathrm{~d} s, \mathrm{~d} z)=\int_{\mathbb{R}^{d}} z^{2} \eta(\mathrm{d} z) \lambda(s) \mathrm{d} s .
$$

Note that by construction, $\|u\|_{\mathcal{L}^{2}(\tilde{\lambda})}=\|\tilde{u}\|_{\mathcal{L}^{2}(v)}$.

Proposition 5. For any $h \in \mathcal{L}^{2}(\tilde{\lambda}), h$ belongs to $\operatorname{dom}\left(\delta^{\widetilde{N}}\right)$ and

$$
\delta^{\tilde{N}}(h)=\left(h^{*}{ }^{(S L)} \widetilde{N}\right)_{T} .
$$

Proof. Since $\delta$ coincides with the Stieltjes-Lebesgue integral with respect to $(\omega-v)$ for predictable processes, we have:

$$
\left.\delta^{\widetilde{N}}(u)=\int_{0}^{T} \int_{\mathbb{R}^{d}} z u(s)(\omega-v)(\mathrm{d} s, \mathrm{~d} z)=\left(u^{*}{ }^{*} \tilde{N}\right)\right)_{T} .
$$

We denote by $D^{\widetilde{N}}$ the adjoint of $\delta^{\widetilde{N}}$.

Proposition 6. We have

$$
D^{\widetilde{N}} F(u)=D F(\tilde{u}) \quad \text { for any } F \in \mathcal{S} .
$$

The associated gradient is equal to:

$$
\nabla^{\widetilde{N}} F=\frac{1}{\int_{\mathbb{R}^{d}} z^{2} \eta(\mathrm{d} z)} \int_{\mathbb{R}^{d}} \nabla_{s, z} F z \eta(\mathrm{d} z) \quad \text { for any } F \in \mathcal{S} .
$$

Proof. The first point comes from the following identity:

$$
\mathbb{E}\left[F \delta^{\widetilde{N}}(u)\right]=\mathbb{E}[F \delta(\tilde{u})]=\mathbb{E}[D F(\tilde{u})] .
$$

For any $F \in \mathcal{S}$, we have:

$$
\begin{aligned}
D^{\widetilde{N}} F(u) & =\int_{0}^{T} \int_{\mathbb{R}^{d}} \nabla_{s, z} F z \eta(\mathrm{d} z) u(s) \lambda(s) \mathrm{d} s=\int_{0}^{T} \frac{1}{\int_{\mathbb{R}^{d}} z^{2} \eta(\mathrm{d} z)} \int_{\mathbb{R}^{d}} \nabla_{s, z} F z \eta(\mathrm{d} z) u(s) \tilde{\lambda}(\mathrm{d} s) \\
& =\left\langle\nabla^{\widetilde{N}} F, u\right\rangle_{\mathcal{L}^{2}(\tilde{\lambda})} \cdot \quad
\end{aligned}
$$

Following the same lines as above, we can introduce, on the space of cylindrical processes, a norm $\|\cdot\|_{2,1}^{\widetilde{N}}$ and extend this space with respect to this norm to get a space denoted by $\mathbb{D}_{2,1}^{\widetilde{N}}\left(\mathcal{H}^{\tilde{\lambda}}\right)$. We can easily prove the next theorem.

\section{Theorem 7.}

(1) $\mathbb{D}_{2,1}^{\tilde{N}}\left(\mathcal{H}^{\tilde{\lambda}}\right) \subset \operatorname{dom}\left(\delta^{\tilde{N}}\right)$.

(2) For any $a \in \mathbb{D}_{2,1}$ and any $\zeta \in \mathbb{D}_{2,1}^{\widetilde{N}}\left(\mathcal{H}^{\tilde{\lambda}}\right)$ we have:

$$
\delta^{\widetilde{N}}(a \zeta)=a \delta^{\widetilde{N}}(\zeta)-\left\langle\nabla^{\widetilde{N}} a ; \zeta\right\rangle_{\mathcal{L}^{2}(\tilde{\lambda})} .
$$


(3) For any $\zeta \in \mathbb{D}_{2,1}^{\widetilde{N}}\left(\mathcal{H}^{\tilde{\lambda}}\right)$ we have:

$$
\mathbb{E}\left[\delta^{\widetilde{N}}(\zeta)^{2}\right]=\mathbb{E}\left[\left\langle\zeta ; \Gamma^{\widetilde{N}} \zeta\right\rangle_{\mathcal{L}^{2}(\tilde{\lambda})}\right]+\mathbb{E}\left[\operatorname{trace}\left(\nabla^{\widetilde{N}} \zeta \circ \nabla^{\widetilde{N}} \zeta\right)\right]
$$

Thus $\mathbb{D}_{2,1}^{\widetilde{N}}\left(\mathcal{H}^{\tilde{\lambda}}\right)$ is included in the $\delta^{\widetilde{N}}$ domain. We now state the relationships between any of the integrals previously defined.

Theorem 8. Let $u \in \mathbb{D}_{2,1}\left(\mathcal{L}^{2}(v)\right)$, then we have:

$$
\delta(u)=\left(u^{(S)}(\omega-v)\right)_{T}-\int_{0}^{T} \int_{\mathbb{R}^{d}} \nabla_{s, z} u_{s, z} v(\mathrm{~d} s, \mathrm{~d} z)
$$

Furthermore, for $u \in \mathbb{D}_{2,1}^{\widetilde{N}}\left(\mathcal{L}^{2}(\tilde{\lambda})\right)$, we have:

$$
\delta^{\tilde{N}}(u)=\left(u^{(S L)} \tilde{N}\right)_{T}-\int_{0}^{T} \nabla_{s}^{\widetilde{N}} u_{s} \tilde{\lambda}(\mathrm{d} s) .
$$

Proof. For the sake of clarity, we will prove only the second point. The first one is analogous. Let $u=\sum_{i=1}^{n} u_{i} \mathbb{I}_{\left(t_{i}, t_{i+1}\right]}$ with $u_{i} \in \mathbb{D}_{2,1}$ and let $\phi \in \mathcal{S}$. Since the Skorohod integral and the Stieltjes-Lebesgue coincides on deterministic processes:

$$
\begin{aligned}
\mathbb{E}\left[\left(u^{(S L)} \tilde{N}\right) \cdot \phi\right] & \left.=\mathbb{E}\left[\sum_{i=1}^{n} u_{i}\left(\mathbb{I}_{\left(t_{i}, t_{i+1}\right]}{ }^{(S L)} \tilde{N}\right) \phi\right]=\sum_{i=1}^{n} \mathbb{E}\left[\delta^{\widetilde{N}}\left(\mathbb{I}_{\left(t_{i}, t_{i+1}\right]}\right) u_{i} \phi\right]=\sum_{i=1}^{n} \mathbb{E}\left[\left\langle\nabla^{\widetilde{N}}\left[u_{i} \phi\right] ; \mathbb{I}_{\left(t_{i}, t_{i+1}\right]}\right)\right\rangle_{\mathcal{L}^{2}(\tilde{\lambda})}\right] \\
& =\sum_{i=1}^{n} \mathbb{E}\left[\left\langle\phi \nabla^{\widetilde{N}} u_{i} ; \mathbb{I}_{\left(t_{i}, t_{i+1}\right]}\right\rangle_{\mathcal{L}^{2}(\tilde{\lambda})}\right]+\sum_{i=1}^{n} \mathbb{E}\left[\left\langle u_{i} \nabla^{\widetilde{N}} \phi ; \mathbb{I}_{\left(t_{i}, t_{i+1}\right]}\right\rangle_{\mathcal{L}^{2}(\tilde{\lambda})}\right]=A_{1}+A_{2} .
\end{aligned}
$$

Moreover,

$$
A_{1}=\mathbb{E}\left[\phi \int_{0}^{T} \nabla_{s}^{\tilde{N}}\left(\sum_{i=1}^{n} u_{i} \mathbb{I}_{\left(t_{i}, t_{i+1}\right]}(s)\right) \tilde{\lambda}(\mathrm{d} s)\right]=\mathbb{E}\left[\phi \int_{0}^{T} \nabla_{s}^{\widetilde{N}} u_{s} \tilde{\lambda}(\mathrm{d} s)\right],
$$

and

$$
A_{2}=\mathbb{E}\left[\left\langle\nabla^{\widetilde{N}} \phi ; \sum_{i=1}^{n} u_{i} \mathbb{I}_{\left(t_{i}, t_{i+1}\right]}\right\rangle_{\mathcal{L}^{2}(\tilde{\lambda})}\right]=\mathbb{E}\left[\phi \delta^{\tilde{N}}\left(\sum_{i=1}^{n} u_{i} \mathbb{I}_{\left(t_{i}, t_{i+1}\right]}\right)\right] .
$$

The equality is true for any $\phi$, so, the result is established for processes of the form $u=\sum_{i=1}^{n} u_{i} \mathbb{I}_{\left(t_{i}, t_{i+1}\right]}$. The general result follows by a limit procedure.

Note that $\nabla_{s}^{\widetilde{N}} u_{s}$ is equal to $0 \mathrm{~d} \mathbb{P} \otimes \tilde{\lambda}(\mathrm{d} s)$ a.s. for $u$ predictable and in $\mathbb{D}_{2,1}\left(\mathcal{L}^{2}(v)\right)$, it is then straightforward that

Theorem 9. The integrals $\delta$ and ${ }_{*}^{(S I)}$ coincide for predictable processes and the integrals $\delta^{\widetilde{N}}, \underset{*}{(S L)}$ and ${ }_{*}^{(S I)}$ coincide for predictable processes.

\subsection{Covariant derivative and Weitzenböck formula}

Consider the space

$$
\mathcal{H}^{\lambda *}=\left\{h \in \mathcal{L}^{2}(\lambda): h^{\prime} \in \mathcal{L}^{2}(\lambda), h(T)=0\right\},
$$


where $h^{\prime}$ denotes the time derivative of $h$, equipped with the scalar product

$$
\langle g, h\rangle_{\mathcal{H}^{\lambda *}}=\langle g, h\rangle_{\mathcal{L}^{2}(\lambda)}+\left\langle g^{\prime}, h^{\prime}\right\rangle_{\mathcal{L}^{2}(\lambda)} .
$$

Introduce the following operators:

$$
\begin{aligned}
& I^{1}: \mathcal{L}^{2} \rightarrow \mathcal{H}^{\lambda *}, \\
& u \mapsto\left(t \mapsto \int_{0}^{t} u(s) \mathrm{d} s\right) \quad \text { and } \quad \begin{aligned}
I^{-1}: \mathcal{H}^{\lambda *} & \rightarrow \mathcal{L}^{2}, \\
u & \mapsto u^{\prime} .
\end{aligned}
\end{aligned}
$$

Definition 6. For $(u, v) \in\left[\mathcal{H}^{\lambda *}\right]^{2}$, we define:

$$
\tilde{\nabla}_{u}(v)=-\frac{1}{\lambda} I^{-1}[v] I^{1}[\lambda u] .
$$

We can write

$$
\tilde{\nabla}_{u}(v)=\langle\tilde{\nabla} .(v), u(\cdot)\rangle_{\mathcal{L}^{2}(\lambda)}
$$

where

$$
\tilde{\nabla}_{s}(v): t \rightarrow \tilde{\nabla}_{s}(v)(t)=-\frac{1}{\lambda(t)} I^{-1}[v](t) \mathbb{I}_{[0, t]}(s) .
$$

Proposition 7. Let $(u, v) \in\left[\mathcal{H}^{\lambda *}\right]^{2}$. We have:

$$
D(\delta(v))(u)=\delta\left(\tilde{\nabla}_{u}(v)\right)+\langle u, v\rangle_{\mathcal{L}^{2}(\lambda)} .
$$

Proof. Consider $q_{u}: s \rightarrow \frac{1}{\lambda(s)} \int_{0}^{s} u(r) \lambda(r) \mathrm{d} r$. We have $v^{\prime} q_{u}=-\tilde{\nabla}_{u}(v)$ and by the very definition of $D$ :

$$
\begin{aligned}
D(\delta(v))(u) & =\int_{0}^{T} \tilde{\nabla}_{u}(v)(s) \omega(\mathrm{d} s)=\left(\tilde{\nabla}_{u}(v)^{(S I)}(\omega-\lambda)\right)_{T}-\int_{0}^{T} v^{\prime}(s) q_{u}(s) \lambda(s) \mathrm{d} s \\
& =\delta\left(\tilde{\nabla}_{u}(v)\right)-\left(\left[v(s) q_{u}(s) \lambda(s)\right]_{0}^{T}-\int_{0}^{T} v(s)\left[q_{u} \lambda\right]^{\prime}(s) \mathrm{d} s\right)=\delta\left(\tilde{\nabla}_{u}(v)\right)+\langle u, v\rangle_{\mathcal{L}^{2}(\lambda)} .
\end{aligned}
$$

Proposition 8. Consider now $F \in \mathcal{S}$ and $(u, v) \in\left[\mathcal{H}^{\lambda *}\right]^{2}$. We have:

$$
D(D F(u))(v)-D(D F(v))(u)=D F\left(\tilde{\nabla}_{v}(u)-\tilde{\nabla}_{u}(v)\right) .
$$

The proof rests on the following lemma.

Lemma 1. Let $(u, v) \in\left[\mathcal{H}^{\lambda *}\right]^{2}$, then

$$
I^{-1}\left[q_{u}\right] q_{v}-I^{-1}\left[q_{v}\right] q_{u}=q_{w},
$$

with

$$
w=\frac{1}{\lambda}\left(I^{1}[\lambda v] I^{-1}[u]-I^{1}[\lambda u] I^{-1}[v]\right)=\tilde{\nabla}_{u}(v)-\tilde{\nabla}_{v}(u) .
$$

Proof. On the one hand, as

$$
I^{-1}\left[q_{u}\right]=-\frac{I^{-1}[\lambda]}{\lambda^{2}} I^{1}[\lambda u]+u,
$$

we have: 


$$
\begin{aligned}
I^{-1}\left[q_{u}\right] q_{v}-I^{-1}\left[q_{v}\right] q_{u} & =-\frac{I^{-1}[\lambda]}{\lambda^{2}} I^{1}[\lambda u] \cdot \frac{1}{\lambda} I^{1}[\lambda v]+\frac{u}{\lambda} I^{1}[\lambda v]+\frac{I^{-1}[\lambda]}{\lambda^{2}} I^{1}[\lambda v] \cdot \frac{1}{\lambda} I^{1}[\lambda u]-\frac{v}{\lambda} I^{1}[\lambda u] \\
& =\frac{1}{\lambda}\left(u I^{1}[\lambda v]-v I^{1}[\lambda u]\right) .
\end{aligned}
$$

On the other hand, letting $g=u I^{1}[\lambda v]-v I^{1}[\lambda u]$ we have:

$$
I^{-1}[g]=I^{-1}[u] I^{1}[\lambda v]+u \lambda v-I^{-1}[v] I^{1}[\lambda u]-v \lambda u=I^{-1}[u] I^{1}[\lambda v]-I^{-1}[v] I^{1}[\lambda u] .
$$

Thus $I^{-1}[g]=\lambda w$ with $w$ defined by (19). Since $g(0)=0$, we can say that $g=I^{1}[\lambda w]$ and $\lambda^{-1} g=q_{w}$ which ends the proof of the lemma.

Proof of Proposition 8. Since $D$ is a derivation operator, it is enough to show the results for an elementary cylindrical variable of the form $F=f\left(\int_{0}^{T} f_{1}(s) \omega(\mathrm{d} s)\right)$. We have:

$$
\begin{aligned}
& D F(u)=-f^{\prime}\left(\int_{0}^{T} f_{1}(s) \omega(\mathrm{d} s)\right) \cdot \int_{0}^{T} f_{1}^{\prime}(s) q_{u}(s) \omega(\mathrm{d} s), \\
& D(D F(u))(v)= f^{\prime \prime}\left(\int_{0}^{T} f_{1}(s) \omega(\mathrm{d} s)\right) \cdot \int_{0}^{T} f_{1}^{\prime}(s) q_{u}(s) \omega(\mathrm{d} s) \cdot \int_{0}^{T} f_{1}^{\prime}(s) q_{v}(s) \omega(\mathrm{d} s) \\
&+f^{\prime}\left(\int_{0}^{T} f_{1}(s) \omega(\mathrm{d} s)\right) \cdot \int_{0}^{T} I^{-1}\left[f_{1}^{\prime} q_{u}\right](s) q_{v}(s) \omega(\mathrm{d} s) .
\end{aligned}
$$

Thus, we have

$$
D(D F(u))(v)-D(D F(v))(u)=+f^{\prime}\left(\int_{0}^{T} f_{1}(s) \omega(\mathrm{d} s)\right) \cdot \int_{0}^{T}\left[I^{-1}\left[f_{1}^{\prime} q_{u}\right](s) q_{v}(s)-I^{-1}\left[f_{1}^{\prime} q_{v}\right](s) q_{u}(s)\right] \omega(\mathrm{d} s) .
$$

Apply Lemma 1, we have:

$$
I^{-1}\left[f_{1}^{\prime} q_{u}\right] q_{v}-I^{-1}\left[f_{1}^{\prime} q_{v}\right] q_{u}=f_{1}^{\prime \prime} q_{u} q_{v}+f_{1}^{\prime} I^{-1}\left[q_{u}\right] q_{v}-f_{1}^{\prime \prime} q_{v} q_{u}-f_{1}^{\prime} q_{u} I^{-1}\left[q_{v}\right]=f_{1}^{\prime} q_{\tilde{\nabla}_{u}(v)-\tilde{\nabla}_{v}(u)} .
$$

The proof is completed if we remark that:

$$
\begin{aligned}
D(D F(u))(v)-D(D F(v))(u) & =+f^{\prime}\left(\int_{0}^{T} f_{1}(s) f_{2}(z) \omega(\mathrm{d} s, \mathrm{~d} z)\right) \cdot \int_{0}^{T} f_{2}(z) f_{1}^{\prime}(s) q_{\tilde{\nabla}_{u}(v)-\tilde{\nabla}_{v}(u)}(s, z) \omega(\mathrm{d} s, \mathrm{~d} z) \\
& =-D F\left(\tilde{\nabla}_{u}(v)-\tilde{\nabla}_{v}(u)\right) .
\end{aligned}
$$

Definition 7. We define the space $\mathcal{S}\left(\mathcal{H}^{\lambda *}\right)$ of cylindrical functionals of the form $F u$ with $F \in \mathcal{S}$ and $u \in \mathcal{H}^{\lambda *}$. Consider $\zeta=F u$ and $\phi=G v$ in $\mathcal{S}\left(\mathcal{H}^{\lambda *}\right)$, it is possible to extend the covariant derivative definition by:

$$
\hat{\nabla}_{u F}(v G)=F v D G(u)+F G \tilde{\nabla}_{u}(v)
$$

and to define the associated gradient still denoted by $\hat{\nabla}$ :

$$
s \rightarrow \hat{\nabla}_{s}(v G)=v \nabla_{s} G+G \tilde{\nabla}_{s}(v)
$$

with $\nabla$ defined by Theorem 3 and $\tilde{\nabla}$ by the relation (16).

Proof. By definition, we have:

$$
\begin{aligned}
\hat{\nabla}_{u F}(v G) & =F v D G(u)+F G \tilde{\nabla}_{u}(v)=F v\left\langle\nabla . G,\left.u(\cdot)\right|_{\mathcal{L}^{2}(\lambda)}+F G\left\langle\tilde{\nabla} . v,\left.u(\cdot)\right|_{\mathcal{L}^{2}(\lambda)}\right.\right. \\
& =\langle v \nabla . G, u(\cdot) F\rangle_{\mathcal{L}^{2}(\lambda)}+\langle G \tilde{\nabla} . v, u(\cdot) F\rangle_{\mathcal{L}^{2}(\lambda)}=\langle v \nabla . G+G \tilde{\nabla} . v, u(\cdot) F\rangle_{\mathcal{L}^{2}(\lambda)}
\end{aligned}
$$

The proof is thus complete. 
Proposition 9. Let $\Phi \in \mathcal{S}$ and $(\zeta, \phi) \in\left[\mathcal{S}\left(\mathcal{H}^{\lambda *}\right)\right]^{2}$. We have:

$$
D(D \Phi(\zeta))(\phi)-D(D \Phi(\phi))(\zeta)=D \Phi\left(\hat{\nabla}_{\phi}(\zeta)-\hat{\nabla}_{\zeta}(\phi)\right) .
$$

Proof. We start with the left-hand side, we can write:

$$
\begin{aligned}
D & (D \Phi(\zeta))(\phi)-D(D \Phi(\phi))(\zeta) \\
& =G D(F D \Phi(u))(v)-F D(G D \Phi(v))(u) \\
& =G F D(D \Phi(u))(v)+G D \Phi(u) D F(v)-F G D(D \Phi(v))(u)-F D \Phi(v) D G(u) \\
& =F G(D(D \Phi(u))(v)-D(D \Phi(v))(u))+G D \Phi(u) D F(v)-F D \Phi(v) D G(u) \\
& =F G D \Phi\left(\tilde{\nabla}_{v}(u)-\tilde{\nabla}_{u}(v)\right)+D \Phi(u G D F(v))-D \Phi(v F D G(u)) \\
& =D \Phi\left(F G\left(\tilde{\nabla}_{v}(u)-\tilde{\nabla}_{u}(v)+u G D F(v)-v F D G(u)\right)\right) \\
& =D \Phi\left(\hat{\nabla}_{v G}(u F)-\hat{\nabla}_{u F}(v G)\right) .
\end{aligned}
$$

The proof is thus complete.

Theorem 10. Let $\zeta \in \mathcal{S}\left(\mathcal{H}^{\lambda *}\right)$, we have:

$$
\mathbb{E}\left[\delta(\zeta)^{2}\right]=\mathbb{E}\left[\|\zeta\|_{\mathcal{L}^{2}(\lambda)}^{2}\right]+\mathbb{E}[\operatorname{trace}(\hat{\nabla}(\zeta) \circ \hat{\nabla}(\zeta))]
$$

Proof. Let $\phi=v G$ and $\zeta=u F$ in $\mathcal{S}\left(\mathcal{H}^{\lambda *}\right)$. Using integration by parts and derivation properties of $D$, we have:

$$
\begin{aligned}
\mathbb{E}[\delta(\zeta) \delta(\phi)]= & \mathbb{E}[F D(\delta(G v))(u)] \\
= & \mathbb{E}[F D(G \delta(v))(u)]-\mathbb{E}[F D(D G(v))(u)] \\
= & \mathbb{E}[F[G D(\delta(v))(u)+\delta(v) D G(u)]]-\mathbb{E}[F D(D G(v))(u)] \\
= & \mathbb{E}\left[F G \delta\left(\tilde{\nabla}_{u}(v)\right)\right]+\mathbb{E}\left[F G\langle u, v\rangle_{\mathcal{L}^{2}(\lambda)}[+\mathbb{E}[F \delta(v) D G(u)]-\mathbb{E}[F D(D G(v))(u)]\right. \\
= & \mathbb{E}\left[\langle\zeta, \phi\rangle_{\mathcal{L}^{2}(\lambda)}\right]+\mathbb{E}\left[D(F G)\left(\tilde{\nabla}_{u}(v)\right)\right]+\mathbb{E}[D(F D G(u))(v)]-\mathbb{E}[F D(D G(v))(u)] \\
= & \mathbb{E}\left[\langle\zeta, \phi\rangle_{\mathcal{L}^{2}(\lambda)}\right]+\mathbb{E}\left[D(F G)\left(\tilde{\nabla}_{u}(v)\right)\right]+\mathbb{E}[D G(u) D F(v)]+\mathbb{E}[F D(D G(u))(v)] \\
& -\mathbb{E}[F D(D G(v))(u)] \\
= & \mathbb{E}\left[\langle\zeta, \phi\rangle_{\mathcal{L}^{2}(\lambda)}\right]+\mathbb{E}\left[F D G\left(\tilde{\nabla}_{u}(v)\right)\right]+\mathbb{E}\left[G D F\left(\tilde{\nabla}_{u}(v)\right)\right]+\mathbb{E}[D G(u) D F(v)] \\
& +\mathbb{E}\left[F D G\left(\tilde{\nabla}_{v}(u)-\tilde{\nabla}_{u}(v)\right)\right] \\
= & \mathbb{E}\left[\langle\zeta, \phi\rangle_{\mathcal{L}^{2}(\lambda)}\right]+\mathbb{E}\left[G D F\left(\tilde{\nabla}_{u}(v)\right)\right]+\mathbb{E}\left[F D G\left(\tilde{\nabla}_{v}(u)\right)\right]+\mathbb{E}[D G(u) D F(v)] .
\end{aligned}
$$

Taking $\phi=\zeta$, we get:

$$
\mathbb{E}\left[\delta(\zeta)^{2}\right]=\mathbb{E}\left[\|\zeta\|_{\mathcal{L}^{2}(\lambda)}\right]+2 \mathbb{E}\left[F D F\left(\tilde{\nabla}_{u}(u)\right)\right]+\mathbb{E}\left[[D F(u)]^{2}\right]=\mathbb{E}\left[\|\zeta\|_{\mathcal{L}^{2}(\lambda)}\right]+B .
$$

The trace term has the following integral expression:

$$
\begin{aligned}
\operatorname{trace}(\hat{\nabla}(\zeta) \circ \hat{\nabla}(\zeta)) & =\int_{0}^{T} \int_{0}^{T} \hat{\nabla}_{s} \zeta(t) \hat{\nabla}_{t} \zeta(s) \lambda(\mathrm{d} s) \lambda(\mathrm{d} t) \\
& =\int_{0}^{T} \int_{0}^{T}\left[u(t) \nabla_{s} F+F \tilde{\nabla}_{s}(u(t))\right]\left[u(s) \nabla_{t} F+F \tilde{\nabla}_{t}(u(s))\right] \lambda(\mathrm{d} s) \lambda(\mathrm{d} t)
\end{aligned}
$$




$$
\begin{aligned}
= & \int_{0}^{T} \int_{0}^{T} u(t) \nabla_{s} F u(s) \nabla_{t} F \lambda(\mathrm{d} s) \lambda(\mathrm{d} t)+2 \int_{0}^{T} \int_{0}^{T} u(t) \nabla_{s} F F \tilde{\nabla}_{t}(u(s)) \lambda(\mathrm{d} s) \lambda(\mathrm{d} t) \\
& +\int_{0}^{T} \int_{0}^{T} F \tilde{\nabla}_{s}(u(t)) F \tilde{\nabla}_{t}(u(s)) \lambda(\mathrm{d} s) \lambda(\mathrm{d} t)=B_{1}+B_{2}+B_{3} .
\end{aligned}
$$

Note, then, that $B_{2}$ is nothing but the first term of $B, B_{1}$ is the second one and $B_{3}$ is equal to zero because $(s, t) \rightarrow$ $\tilde{\nabla}_{s}(u(t)) \tilde{\nabla}_{t}(u(s))$ is non-null on a $\lambda \otimes \lambda$ negligible set.

As in the previous section, we define, for $\phi \in \mathcal{S}\left(\mathcal{H}^{\lambda *}\right)$ the following norm:

$$
\left[\|\phi\|_{2,1}^{C}\right]^{2}=\mathbb{E}\left[\|\phi\|_{\mathcal{L}^{2}(\lambda)}^{2}\right]+\mathbb{E}\left[\|\hat{\nabla} \phi\|_{\mathcal{L}^{2}(\lambda) \otimes \mathcal{L}^{2}(\lambda)}^{2}\right],
$$

and we consider $\mathbb{D}_{2,1}^{C}\left(\mathcal{H}^{\lambda *}\right)$ the closure of $\mathcal{S}\left(\mathcal{H}^{\lambda *}\right)$ with respect to this norm. The same demonstration as that of Proposition 4 implies that $\mathbb{D}_{2,1}^{C}\left(\mathcal{H}^{\lambda *}\right)$ is a subset of $\operatorname{dom}(\delta)$. Following the ideas of Privault $[18,17,19]$ we can introduce exterior derivative, for $\phi \in \mathcal{S}\left(\mathcal{H}^{\lambda *}\right)$ by:

$$
\mathrm{d} \phi(s, t)=\frac{1}{2}\left(\hat{\nabla}_{s} \phi(t)-\hat{\nabla}_{t} \phi(s)\right) \text { for all }(s, t) \in\left[\mathbb{R}^{+}\right]^{2}
$$

and

$$
\left\langle\mathrm{d} \phi, h_{1} \wedge h_{2}\right\rangle_{\mathcal{L}^{2}(\lambda) \wedge \mathcal{L}^{2}(\lambda)}=\frac{1}{2}\left(\left\langle\hat{\nabla}_{h_{1}} \phi, h_{2}\right\rangle_{\mathcal{L}^{2}(\lambda)}-\left\langle\hat{\nabla}_{h_{2}} \phi, h_{1}\right\rangle_{\mathcal{L}^{2}(\lambda)}\right),
$$

thus

$$
\|\mathrm{d} \phi\|_{\mathcal{L}^{2}(\lambda) \wedge \mathcal{L}^{2}(\lambda)}=\frac{1}{2} \int_{0}^{T} \int_{0}^{T}\left(\hat{\nabla}_{s} \phi(t)-\hat{\nabla}_{t} \phi(s)\right)^{2} \lambda(\mathrm{d} s) \lambda(\mathrm{d} t) .
$$

We can now state a Weitzenböck type formula:

Theorem 11. Let $\zeta \in \mathcal{S}\left(\mathcal{H}^{\lambda *}\right)$, we have:

$$
\mathbb{E}\left[\delta(\zeta)^{2}\right]+\mathbb{E}\left[\|\mathrm{d} \zeta\|_{\mathcal{L}^{2}(\lambda) \wedge \mathcal{L}^{2}(\lambda)}^{2}\right]=\mathbb{E}\left[\|\zeta\|_{\mathcal{L}^{2}(\lambda)}^{2}\right]+\mathbb{E}\left[\|\hat{\nabla}(\zeta)\|_{\mathcal{L}^{2}(\lambda)}^{2}\right]
$$

Proof. Follows from Theorem 10 by polarization.

\section{Stochastic calculus for Filtered Poisson Processes}

\subsection{Filtered Marked Poisson Processes}

Let $K$ be a deterministic kernel and define, when it exists, the following process:

$$
N_{t}^{K}=\int_{0}^{t} K(t, s) \mathrm{d} N_{s}:=\int_{0}^{t} \int_{\mathbb{R}^{d}} z K(t, s) \omega(\mathrm{d} s, \mathrm{~d} z) .
$$

We call it Filtered Poisson Process and its compensated version is defined by:

$$
\widetilde{N}_{t}^{K}:=\int_{0}^{t} \int_{\mathbb{R}^{d}} z K(t, s)(\omega-v)(\mathrm{d} s, \mathrm{~d} z) .
$$

If $\left\{\left(T_{n}, Z_{n}\right): n \in \mathbb{N}\right\}$ denote impact times and corresponding marks of $N$, we can write:

$$
N_{t}^{K}=\sum_{n \geqslant 1} K\left(t, T_{n}\right) Z_{n} \mathbb{I}_{\left[T_{n} \leqslant t\right]} .
$$




\section{Hypothesis 1.}

(1) The kernel $(s, z) \rightarrow z K(t, s)$ belongs to $\mathcal{L}^{2}(v)$ for any $t>0$.

(2) $K$ is triangular in the following way:

$$
K(t, s)=0 \text { for all } s>t>0 .
$$

(3) $K$ "does not explode on the diagonal":

$$
K(t, t)<\infty \text { for all } t \geqslant 0 .
$$

(4) For any $t \geqslant 0$ the function:

$$
\begin{aligned}
K(t, \cdot):[0, t[ & \rightarrow \mathbb{R} \quad \text { is càdlàg, } \\
s & \rightarrow K(t, s) .
\end{aligned}
$$

(5) For any $s \geqslant 0$ the function:

$$
\begin{aligned}
K(\cdot, s):[s, T[ & \rightarrow \mathbb{R} \text { has bounded variations, } \\
t & \rightarrow K(t, s) .
\end{aligned}
$$

Note that under Hypothesis 1 , the processes $N^{K}$ and $\widetilde{N}^{K}$ are well defined, with càdlàg sample-paths and $N^{K}$ has finite variation on $[0, T]$.

Example 1. From the expression (24), one can note that this class of processes includes "Shot Noise processes", which corresponds to a convolution kernel $K(t, s)=k(t-s)$.

Example 2. One can also take the kernel defining the fractional Brownian motion: $K^{(H)}$. This process will be called fractional Poisson process. This process is a fractional Lévy motion, a class of processes defined in [2]. Note that the third point of Hypothesis 1 excludes the case of the kernel $K^{(H)}$ with $H<\frac{1}{2}$.

Example 3. One can also introduce an "Ornstein-Uhlenbeck process" by considering the kernel:

$$
(s, t) \rightarrow \mathrm{e}^{\alpha(t-s)}
$$

where $\alpha$ is a positive constant. It will be denoted by $N^{(\mathrm{OU})}$.

The following proposition is clear.

Proposition 10. $N^{K}$ is not a marked point process. If $K$ is continuous then $N^{K}$ has the same jump times as $N$ and if $K$ is null on the diagonal and if $K$ is continuous, then $N^{K}$ is continuous.

Theorem 12. The following results hold for any $t \in[0, T]$ and any $t^{\prime} \in[0, T]$ :

$$
\begin{aligned}
& \mathbb{E}\left[\widetilde{N}_{t}^{K}\right]=0, \\
& \mathbb{E}\left[\left(\widetilde{N}_{t}^{K}\right)^{2}\right]=\int_{0}^{t} \int_{\mathbb{R}^{d}} z^{2} K(t, s)^{2} v(\mathrm{~d} s, \mathrm{~d} z)=\int_{0}^{t} K(t, s)^{2} \tilde{\lambda}(\mathrm{d} s), \\
& \operatorname{Cov}\left(\widetilde{N}_{t}^{K} ; \widetilde{N}_{t^{\prime}}^{K}\right):=\int_{0}^{t \wedge t^{\prime}} \int_{\mathbb{R}^{d}} z^{2} K(t, s) K\left(t^{\prime}, s\right) v(\mathrm{~d} s, \mathrm{~d} z) \int_{0}^{t \wedge t^{\prime}} K(t, s) K\left(t^{\prime}, s\right) \tilde{\lambda}(\mathrm{d} s) .
\end{aligned}
$$

These formulas are known as the Campbell's formulas. However, one can give a new proof by using the following lemma which is a very interesting result in itself: 
Lemma 2. If for any $r \in[0, T]$ we have:

$$
\iint_{0}^{T} z_{\mathbb{R}^{d}} z^{2} K^{2}(r, s) v(\mathrm{~d} s, \mathrm{~d} z)<\infty
$$

Then, for every fixed $r \in[0, T]$, the process:

$$
\left\{M_{t}^{r}:=\int_{0}^{t} \int_{\mathbb{R}^{d}} z K(r, s)(\omega-v)(\mathrm{d} s, \mathrm{~d} z), t \geqslant 0\right\}
$$

is a $\left(\mathfrak{F}_{t}\right)$-martingale with the following quadratic variation:

$$
\left\langle M^{r}, M^{r}\right\rangle_{t}:=\int_{0}^{t} \int_{\mathbb{R}^{d}} z^{2} K^{2}(r, s) v(\mathrm{~d} s, \mathrm{~d} z) \quad \text { for all } t \in[0, T] .
$$

Proof. A time $r$ fixed, we are in the presence of a stochastic integral relative with the martingale measure $(\omega-v)$ and thus in the presence of a martingale.

Proof of Theorem 12. The first equality is the definition of $\widetilde{N}$. For the second one, as $M^{r}$ is a $\left(\mathfrak{F}_{t}\right)$-martingale, we have:

$$
\mathbb{E}\left[\left(M_{t}^{r}\right)^{2}\right]=\mathbb{E}\left[\left\langle M^{r}, M^{r}\right\rangle_{t}\right]:=\int_{0}^{t} \int_{\mathbb{R}^{d}} z^{2} K^{2}(r, s) v(\mathrm{~d} s, \mathrm{~d} z) .
$$

It is now enough to take $r=t$ to get:

$$
\mathbb{E}\left[\left(M_{t}^{t}\right)^{2}\right]=\mathbb{E}\left[\left(N_{t}^{K}\right)^{2}\right]:=\int_{0}^{t} \int_{\mathbb{R}^{d}} z^{2} K^{2}(t, s) v(\mathrm{~d} s, \mathrm{~d} z) .
$$

The third one follows by polarization.

Theorem 13. $N^{K}$ is a semi-martingale but not a martingale.

Proof. It is a semi-martingale because it is a finite variation on compacts process. It is not a martingale because it is continuous (in the case $K$ null on the diagonal).

Theorem 14. For any function $f$ locally bounded, it is possible to define, in Stieltjes-Lebesgue way, the following processes:

$$
\left\{\left(f^{(\text {(SL) }} N^{K}\right)_{t}: t \in \mathbb{R}^{+}\right\} .
$$

Proof. The process $N^{K}$ being of finite variation. The integral is well defined in Stieltjes-Lebesgue way.

Theorem 15. There exists an application $\mathcal{K}^{*}: \mathcal{L}^{2}(\tilde{\lambda}) \rightarrow \mathcal{L}^{2}(\tilde{\lambda})$ linear, continuous, satisfying $\mathcal{K}^{*}\left(\mathbb{I}_{[0, t]}\right)=K(t, \cdot)$ and, for any $f \in \mathcal{L}^{2}(\tilde{\lambda})$ locally bounded,

$$
\left(f^{*}{ }^{(S L)} \widetilde{N}^{K}\right)_{t}=\left(\mathcal{K}^{*}(f)^{*} \stackrel{(S L)}{N}\right)_{t} \text { for all } t \in \mathbb{R}^{+} .
$$

Proof. For any $f \in \mathcal{L}^{2}(\tilde{\lambda})$, we have the following relationship:

$$
\|f\|_{\mathcal{L}^{2}(\mathrm{~d} t)} \geqslant c\|f\|_{\mathcal{L}^{2}(\tilde{\lambda})} .
$$


In fact, by definition we have:

$$
\tilde{\lambda}(\mathrm{d} s)=\int_{\mathbb{R}^{d}} z^{2} \eta(\mathrm{d} z) \lambda(s) \mathrm{d} s,
$$

thus using the lower-boundedness assumption on $\lambda$ we can write:

$$
\int_{0}^{T} f^{2}(s) \tilde{\lambda}(\mathrm{d} s)=\int_{\mathbb{R}^{d}} z^{2} \eta(\mathrm{d} z) \int_{0}^{T} f^{2}(s) \lambda(s) \mathrm{d} s \geqslant m \int_{\mathbb{R}^{d}} z^{2} \eta(\mathrm{d} z) \int_{0}^{T} f^{2}(s) \mathrm{d} s
$$

and (25) follows. Now, let us introduce the following operator $K$ :

$$
\begin{aligned}
K: \mathcal{L}^{2}(\tilde{\lambda}) & \rightarrow \mathcal{L}^{2}(\mathrm{~d} t), \\
f & \rightarrow \int_{0}^{T} K(t, s) f(s) \tilde{\lambda}(\mathrm{d} s) .
\end{aligned}
$$

It is known [7] that $K$ is a continuous operator from $\mathcal{L}^{2}(\mathrm{~d} t)$ in $\mathcal{L}^{2}(\mathrm{~d} t)$, according to (25) it is also continuous from $\mathcal{L}^{2}(\tilde{\lambda})$ into $\mathcal{L}^{2}(\mathrm{~d} t)$. Consider now the operator:

$$
\begin{aligned}
I_{T-}^{T}: \mathcal{L}^{2}(\tilde{\lambda}) & \rightarrow \mathcal{L}^{2}(\mathrm{~d} t), \\
f & \rightarrow \int f(s) \mathrm{d} s .
\end{aligned}
$$

We have:

$$
\left\|I_{T-}^{T}(f)\right\|_{\mathcal{L}^{2}(\mathrm{~d} t)}^{2}=\int_{0}^{T}\left(\int_{S}^{T} f(u) \mathrm{d} u\right)^{2} \mathrm{~d} s \leqslant \int_{0}^{T} \int_{0}^{T} f^{2}(u) \mathrm{d} u \mathrm{~d} s \leqslant c \int_{0}^{T} f^{2}(u) \lambda(u) \mathrm{d} u \leqslant c\|f\|_{\mathcal{L}^{2}(\tilde{\lambda})}^{2}
$$

thus $I_{T-}^{T}$ is continuous from $\mathcal{L}^{2}(\tilde{\lambda})$ into $\mathcal{L}^{2}(\mathrm{~d} t)$, moreover, its adjoint is given by:

$$
\begin{aligned}
I_{0+}: \mathcal{L}^{2} & \rightarrow \mathcal{L}^{2}(\tilde{\lambda}), \\
f & \rightarrow \frac{1}{\lambda(\cdot)} \int_{0} f(s) \mathrm{d} s .
\end{aligned}
$$

In fact, we have:

$$
\begin{aligned}
\left\langle f, I_{0+}(g)\right\rangle_{\mathcal{L}^{2}(\tilde{\lambda})} & =\int_{0}^{T} f(s) \frac{1}{\tilde{\lambda}(s)} \int_{0}^{s} g(t) \mathrm{d} t \tilde{\lambda}(s) \mathrm{d} s=\int_{0}^{T} f(s) \int_{0}^{T} g(t) \mathbb{I}_{[0, s]}(t) \mathrm{d} t \mathrm{~d} s \\
& =\int_{0}^{T} \int_{0}^{T} f(s) g(t) \mathbb{I}_{[t, T]}(s) \mathrm{d} t \mathrm{~d} s=\int_{0}^{T} g(t) \int_{t}^{T} f(s) \mathrm{d} s \mathrm{~d} t=\left\langle g, I_{T-}^{T}(f)\right\rangle_{\mathcal{L}^{2}} .
\end{aligned}
$$

Then, we introduce for $g \in \mathcal{L}^{2}(\tilde{\lambda})$, the linear form:

$$
\begin{aligned}
\theta_{g}: \mathcal{L}^{2}(\tilde{\lambda}) & \rightarrow \mathbb{R}, \\
f & \rightarrow \int_{0}^{T} g(s) K(f)(s) \mathrm{d} s .
\end{aligned}
$$

Using the Cauchy-Schwartz inequality and the relation (5), we have: 


$$
\left|\int_{0}^{T} g(s) K(f)(s) \mathrm{d} s\right| \leqslant\|g\|_{\mathcal{L}^{2}(\mathrm{~d} t)}\|K(f)\|_{\mathcal{L}^{2}(\mathrm{~d} t)} \leqslant c_{4}\|g\|_{\mathcal{L}^{2}(\mathrm{~d} t)}\|f\|_{\mathcal{L}^{2}(\tilde{\lambda})} \leqslant c\|f\|_{\mathcal{L}^{2}(\tilde{\lambda})} .
$$

The linear application is thus continuous, so there exists a continuous operator $K^{*}$ such that:

$$
\theta_{g}=\left\langle K^{*}(g), f\right\rangle_{\mathcal{L}^{2}(\tilde{\lambda})},
$$

this means:

$$
\int_{0}^{T} g(s) K(f)(s) \mathrm{d} s=\int_{0}^{T} K^{*}(g)(s) f(s) \tilde{\lambda}(s) \mathrm{d} s .
$$

For any $g \in \mathcal{L}^{2}(\tilde{\lambda})$, using Fubini in the third equality, we get:

$$
\begin{aligned}
\left\langle K^{*} f, g\right\rangle_{\mathcal{L}^{2}} & =\int_{0}^{T} f(s) \int_{0}^{T} K(s, v) g(v) \tilde{\lambda}(v) \mathrm{d} v \mathrm{~d} s=\int_{0}^{T} \int_{0}^{T} f(s) K(s, v) g(v) \tilde{\lambda}(v) \mathrm{d} s \mathrm{~d} v \\
& =\int_{0}^{T}\left[\int_{0}^{T} f(s) K(s, v) \mathrm{d} s\right] g(v) \tilde{\lambda}(v) \mathrm{d} v .
\end{aligned}
$$

Thus,

$$
\begin{aligned}
K^{*}: \mathcal{L}^{2} & \rightarrow \mathcal{L}^{2}(\tilde{\lambda}), \\
f & \rightarrow \int_{0}^{T} K(s, \cdot) f(s) \mathrm{d} s .
\end{aligned}
$$

Finally, we define the operator:

$$
\begin{aligned}
\mathcal{K}^{*}: \mathcal{L}^{2}(\tilde{\lambda}) & \rightarrow \mathcal{L}^{2}(\tilde{\lambda}), \\
f & \rightarrow K^{*} \circ\left[I_{T^{-}}^{T}\right]^{-1}(f) .
\end{aligned}
$$

$\mathcal{K}^{*}$ is linear and continuous from $\mathcal{L}^{2}(\tilde{\lambda})$ to $\mathcal{L}^{2}(\tilde{\lambda})$. It is then easy to show that

$$
\mathcal{K}^{*}\left(\mathbb{I}_{[0, t]}\right)=K(t, \cdot) .
$$

Let us now show that

$$
\left(f * \widetilde{N}^{K}\right)=\left(\mathcal{K}^{*}(f)^{*} \stackrel{(S L)}{N}\right) \quad \text { for all } f \in \mathcal{L}^{2}(\tilde{\lambda})
$$

For any $t \in[0, T]$, we have:

$$
\left(\mathbb{I}_{[0, t]} * \widetilde{N}^{K}\right)_{t}=\widetilde{N}_{t}^{K}=\left(\mathbb{I}_{[0, t]}{ }^{*}{ }^{(S L)} \widetilde{N}^{K}\right)_{t} .
$$

In fact,

$$
\left(\mathbb{I}_{[0, t]} * \widetilde{N}^{K}\right)_{t}=\left(\mathcal{K}^{*}\left(\mathbb{I}_{[0, t]}\right)^{*}{ }^{(S L)} \tilde{N}\right)_{t}=\left(K(t, \cdot)\left(\mathbb{I}_{[0, t]}\right)^{*} \stackrel{(S L)}{*}\right)_{t}=\widetilde{N}_{t}^{K}=\left(\mathbb{I}_{[0, t]}{ }^{*}{ }^{(S L)} \widetilde{N}^{K}\right)_{t} .
$$

It is then routine to extend this result to any $f \in \mathcal{L}^{2}(\tilde{\lambda})$.

We now define the vector space

$$
\mathcal{I}=\operatorname{span}\left\{\mathbb{I}_{[0, t]}: t \in[0, T]\right\},
$$

equipped with the following scalar product:

$$
\left\langle\mathbb{I}_{[0, t]} ; \mathbb{I}_{[0, s]}\right\rangle_{\mathcal{I}^{K}}=\langle K(t, \cdot) ; K(s, \cdot)\rangle_{\mathcal{L}^{2}(\tilde{\lambda})} .
$$

Finally, let $\mathcal{I}^{K}$ be the closure of $\mathcal{I}$ with respect to this inner product. 
Theorem 16. $\mathcal{K}^{*}$ is an isometry from $\mathcal{I}^{K}$ into $\mathcal{L}^{2}(\tilde{\lambda})$ and for any $f \in \mathcal{I}^{K}, g \in \mathcal{I}^{K}$ we have:

$$
\mathbb{E}\left[\left(f^{(S L)} \tilde{N}^{K}\right)_{T} \cdot\left(g^{(S L)} \tilde{N}^{K}\right)_{T}\right]=\langle f, g\rangle_{\mathcal{I}^{K}} .
$$

Proof. For any $t \in[0, T]$, we have:

$$
\left\|\mathbb{I}_{[0, t]}\right\|_{\mathcal{I}^{K}}=\|K(t, \cdot)\|_{\mathcal{L}^{2}(\tilde{\lambda})}=\left\|\mathcal{K}^{*}\left(\mathbb{I}_{[0, t]}\right)\right\|_{\mathcal{L}^{2}(\tilde{\lambda})} .
$$

The isometry identity is thus satisfied by elements of $\mathcal{I}$. Since $\mathcal{K}^{*}$ is continuous, the result holds for elements of $\mathcal{I}^{K}$. The second point follows from the following computations:

$$
\begin{aligned}
\mathbb{E}\left[\left(f^{*}{ }^{(S L)} \widetilde{N}^{K}\right)_{T} \cdot\left(g^{*}{ }^{(S L)} \widetilde{N}^{K}\right)_{T}\right] & =\mathbb{E}\left[\left(\mathcal{K}^{*}(f)^{*} \stackrel{(S L)}{N}\right)_{T} \cdot\left(\mathcal{K}^{*}(g)^{*} \stackrel{(S L)}{N}\right)_{T}\right] \\
& =\left\langle\mathcal{K}^{*}(f), \mathcal{K}^{*}(g)\right\rangle_{\mathcal{L}^{2}(\tilde{\lambda})}=\langle f, g\rangle_{\mathcal{I}^{K}} .
\end{aligned}
$$

Theorem 17. The processes $\widetilde{N}^{K}$ and $\tilde{N}$ have the same filtrations.

Proof. On the one hand, for any $t \in[0, T]$, there exists $\left(\alpha_{i}, t_{i}\right) \in(\mathbb{R} \times[0, T])^{\mathbb{N}}$ such that:

$$
K(t, \cdot)=\mathcal{L}_{n \rightarrow \infty}^{2}-\lim \sum_{i=1}^{n} \alpha_{i} \mathbb{I}_{\left[0, t_{i}\right]} .
$$

On the other hand, for any $s \in[0, T]$, there exists $\left(\beta_{i}, s_{i}\right) \in(\mathbb{R} \times[0, T])^{\mathbb{N}}$ such that:

$$
\mathbb{I}_{[0, s]}=\mathcal{L}_{n \rightarrow \infty}^{2}-\lim _{i=1} \sum_{i}^{n} \beta_{i} K\left(s_{i}, \cdot\right) .
$$

The family $\left\{K\left(t_{i}, \cdot\right)\right\}$ is complete as image of the complete family $\left\{\varepsilon_{t_{i}}\right\}$ by the injective application $K$. From (27), we deduce that

$$
\begin{aligned}
& \left(K(t, \cdot)^{(S L)} \tilde{N}\right)=\underset{n \rightarrow \infty}{\mathbb{L}^{2}(\mathbb{P})-\lim } \sum_{i=1}^{n} \alpha_{i}\left(\mathbb{I}_{\left[0, t_{i}\right]}{ }^{(S L)} \tilde{N}\right), \\
& \widetilde{N}_{t}^{K}=\underset{n \rightarrow \infty}{\mathbb{L}^{2}(\mathbb{P})-\lim } \sum_{i=1}^{n} \alpha_{i} \widetilde{N}_{t_{i}} .
\end{aligned}
$$

Thus, $\widetilde{N}_{t}^{K} \in \mathfrak{F}_{t}$ which shows the first inclusion. Now, from (28):

$$
\begin{aligned}
& \left(\mathbb{I}_{[0, s]}{ }^{*}{ }^{(S L)} \tilde{N}\right)=\mathbb{L}^{2}(\mathbb{P})-\lim \sum_{i=1}^{n} \beta_{i}\left(K\left(s_{i}, \cdot\right)^{(S L)} \tilde{N}\right), \\
& \widetilde{N}_{s}=\mathbb{L}^{2}(\mathbb{P})-\lim \sum_{i=1}^{n} \beta_{i} \widetilde{N}_{s_{i}}^{K} .
\end{aligned}
$$

It follows that $\widetilde{N}_{t}$ belongs to $\mathfrak{F}_{t}^{K}$ and the result follows.

We now turn to the definition for the Skorohod-like integral with respect to marked point processes.

Definition 8. For any $u$ such that $\mathcal{K}^{*}(u) \in \operatorname{dom}\left(\delta^{\tilde{N}}\right)$ we define the integral with respect to $\widetilde{N}^{K}$ by:

$$
\delta^{\widetilde{N}^{K}}(u)=\delta^{\widetilde{N}}\left(\mathcal{K}^{*}(u)\right)=\delta\left(z \cdot \mathcal{K}^{*}(u)\right)
$$

Definition 9. For any $F \in \mathcal{S}$, for any $h \in \mathcal{L}^{2}(\tilde{\lambda})$ we define the directional derivative by: 


$$
\begin{aligned}
D^{\widetilde{N}^{K}} F(h)= & D^{\widetilde{N}} F\left(\mathcal{K}^{*}(h)\right)=D F\left(z \cdot \mathcal{K}^{*}(h)\right) \\
= & -\sum_{i=1}^{n} \frac{\partial f}{\partial x_{i}}\left(\iint_{0} f_{\mathbb{R}^{d}}(s) g_{1}(z) \omega(\mathrm{d} s, \mathrm{~d} z), \ldots, \iint_{0} f_{\mathbb{R}^{d}}(s) g_{n}(z) \omega(\mathrm{d} s, \mathrm{~d} z)\right) \\
& \times \int_{0}^{T} \int_{\mathbb{R}^{d}} f_{i}^{\prime}(s) g_{i}(z)\left(\frac{z}{\lambda(s)} \int_{0}^{s} \mathcal{K}^{*}(h)(r) \lambda(r) \mathrm{d} r\right) \omega(\mathrm{d} s, \mathrm{~d} z) .
\end{aligned}
$$

The following properties motivate the previous definition:

Proposition 11. For any $F, G \in \mathcal{S}$ and any $h \in \mathcal{L}^{2}(\tilde{\lambda})$ we have:

$$
\begin{aligned}
& D^{\widetilde{N}^{K}}(F G)(h)=F \cdot D^{\widetilde{N}^{K}} G(h)+G \cdot D^{\widetilde{N}^{K}} F(h), \\
& \mathbb{E}\left[D^{\widetilde{N}^{K}} F(h)\right]=\mathbb{E}\left[F \cdot \delta^{\widetilde{N}^{K}}(h)\right] .
\end{aligned}
$$

Proof. Let $F, G \in \mathcal{S}$ and $h \in \mathcal{L}^{2}(\tilde{\lambda})$ we have:

$$
\begin{aligned}
D^{\widetilde{N}^{K}}(F G)(h) & =D^{\widetilde{N}}(F G)\left(\mathcal{K}^{*}(h)\right)=F \cdot D^{\widetilde{N}}(G)\left(\mathcal{K}^{*}(h)\right)+G \cdot D^{\widetilde{N}}(F)\left(\mathcal{K}^{*}(h)\right) \\
& =F \cdot D^{\widetilde{N}^{K}} G(h)+G \cdot D^{\widetilde{N}^{K}} F(h), \\
\mathbb{E}\left[D^{\widetilde{N}^{K}} F(h)\right] & =\mathbb{E}\left[D^{\widetilde{N}} F\left(\mathcal{K}^{*}(h)\right)\right]=\mathbb{E}\left[F \cdot \delta^{\widetilde{N}}\left(\mathcal{K}^{*}(h)\right)\right]=\mathbb{E}\left[F \cdot \delta^{\widetilde{N}^{K}}(h)\right] .
\end{aligned}
$$

Proposition 12. For any $F \in \mathcal{S}$, the $\mathcal{L}^{2}(\tilde{\lambda})$-valued random variable, $\nabla^{\widetilde{N}^{K}}(F)$, defined by

$$
\nabla^{\tilde{N}^{K}}(F)=\mathcal{K} \circ \nabla^{\tilde{N}}(F),
$$

satisfies

$$
D^{\widetilde{N}^{K}} F(h)=\left\langle\nabla^{\widetilde{N}^{K}}(F), h\right\rangle_{\mathcal{L}^{2}(\tilde{\lambda})} .
$$

We call $\nabla^{\widetilde{N}^{K}}$ the gradient map associated to $D^{\widetilde{N}^{K}}$.

Proof. Let $F \in \mathcal{S}$ and $h \in \mathcal{L}^{2}(\tilde{\lambda})$, we have:

$$
D^{\widetilde{N}^{K}} F(h)=D^{\widetilde{N}}(F)\left(\mathcal{K}^{*}(h)\right)=\left\langle\nabla^{\widetilde{N}} F ;\left.\mathcal{K}^{*}(h)\right|_{\mathcal{L}^{2}(\tilde{\lambda})}=\left\langle\mathcal{K} \circ \nabla^{\widetilde{N}} F ; h\right\rangle_{\mathcal{L}^{2}(\tilde{\lambda})} .\right.
$$

It is obvious that this gradient satisfies the properties of derivation and integration by parts. Moreover, it is the adjoint operator of $\delta^{\widetilde{N}^{K}}$ and it follows from Proposition 11 that the mapping $F \rightarrow \nabla^{\widetilde{N}^{K}} F$ is closable in $\mathbb{L}^{2}(\mathbb{P})$. Consider the following space:

$$
\mathcal{H}^{K}=\left\{\zeta \in \mathcal{L}^{2}(\tilde{\lambda}): \mathcal{K}^{*}(\zeta) \in \mathcal{H}^{\tilde{\lambda}}\right\}
$$

With notations now classical, we define on $\mathcal{S}\left(\mathcal{H}^{K}\right)$ the operator $\Gamma^{K}$ by:

$$
\begin{aligned}
\Gamma^{K}: S\left(\mathcal{H}^{K}\right) & \rightarrow \mathbb{L}^{2}(\Omega \times[0, T], \mathrm{d} \mathbb{P} \otimes \tilde{\lambda}), \\
\zeta & \mapsto \mathcal{K} \circ \Gamma^{\widetilde{N}} \circ \mathcal{K}^{*}(\zeta)
\end{aligned}
$$

and we set

$$
\|\Phi\|_{2,1}^{\Gamma^{K}}=\frac{1}{2}\left(\mathbb{E}\left[\|\Phi\|_{\mathcal{L}^{2}(\tilde{\lambda})}\right]+\mathbb{E}\left[\left\|\Gamma^{K} \Phi\right\|_{\mathcal{L}^{2}(\tilde{\lambda})}\right]\right)+\mathbb{E}\left[\left\|\nabla^{\widetilde{N}}\left(\mathcal{K}^{*} \Phi\right)\right\|_{\mathcal{L}^{2}(\tilde{\lambda}) \otimes \mathcal{L}^{2}(\tilde{\lambda})}^{2}\right] .
$$

Consider $\mathbb{D}_{2,1}^{K}\left(\mathcal{H}^{K}\right)$ the closure of $\mathcal{S}\left(\mathcal{H}^{K}\right)$ with respect to this norm. We, of course, have

$$
\mathbb{D}_{2,1}^{K}\left(\mathcal{H}^{K}\right) \subset \operatorname{dom}\left(\delta^{\tilde{N}^{K}}\right) .
$$


Theorem 18. For any $a \in \mathbb{D}_{2,1}$ and any $\zeta \in \mathbb{D}_{2,1}^{K}\left(\mathcal{H}^{K}\right)$ we have:

$$
\delta^{\widetilde{N}^{K}}(a \zeta)=a \delta^{\widetilde{N}^{K}}(\zeta)-\left\langle\nabla^{\widetilde{N}^{K}} a ; \zeta\right\rangle_{\mathcal{L}^{2}(\tilde{\lambda})}
$$

and for any $\zeta \in \mathbb{D}_{2,1}^{K}\left(\mathcal{H}^{K}\right)$ we have:

$$
\mathbb{E}\left[\delta^{\widetilde{N}^{K}}(\zeta)^{2}\right]=\mathbb{E}\left[\left\langle\zeta ; \Gamma^{K} \zeta\right\rangle_{\mathcal{L}^{2}(\tilde{\lambda})}\right]+\mathbb{E}\left[\operatorname{trace}\left(\nabla^{\widetilde{N}}\left(\mathcal{K}^{*} \zeta\right) \circ \nabla^{\widetilde{N}}\left(\mathcal{K}^{*} \zeta\right)\right)\right]
$$

Proof. Apply Theorem 7 to $\mathcal{K}^{*} \zeta$.

Theorem 19. Let $u \in \mathbb{D}_{2,1}^{K}\left(\mathcal{L}^{2}([0, T])\right)$, then, if the different terms are defined, we get the following equality:

$$
\delta^{\tilde{N}^{K}}(u)=\left(u^{(S L)} \tilde{N}^{K}\right)_{T}-\int_{0}^{T} \nabla_{s}^{\tilde{N}^{K}} u_{s} \tilde{\lambda}(\mathrm{d} s) .
$$

Proof. Let $u=\sum_{i=1}^{n} u_{i} \mathbb{I}_{\left(t_{i}, t_{i+1}\right]}$ with $u_{i} \in \mathbb{D}_{2,1}^{K}$ and let $\phi \in \mathcal{S}$, we have:

$$
\begin{aligned}
& \mathbb{E}\left[\phi\left(u^{(S L)} \widetilde{N}^{K}\right)_{T}\right]=\sum_{i=1}^{n} \mathbb{E}\left[\left(\left(\mathbb{I}_{\left(t_{i}, t_{i+1}\right]}{ }^{(S L)} \widetilde{N}^{K}\right)_{T}\right) u_{i} \phi\right]=\sum_{i=1}^{n} \mathbb{E}\left[\delta^{\widetilde{N}^{K}}\left(\mathbb{I}_{\left(t_{i}, t_{i+1}\right]}\right) u_{i} \phi\right] \\
& =\sum_{i=1}^{n} \mathbb{E}\left[\left\langle\nabla^{\widetilde{N}^{K}}\left[u_{i} \phi\right] ; \mathbb{I}_{\left(t_{i}, t_{i+1}\right]}\right\rangle_{\mathcal{L}^{2}(\tilde{\lambda})}\right] \\
& =\sum_{i=1}^{n} \mathbb{E}\left[\left\langle\phi \nabla^{\widetilde{N}^{K}}\left[u_{i}\right] ; \mathbb{I}_{\left(t_{i}, t_{i+1}\right]}\right\rangle_{\mathcal{L}^{2}(\tilde{\lambda})}\right]+\sum_{i=1}^{n} \mathbb{E}\left[\left\langle u_{i} \nabla^{\widetilde{N}^{K}}[\phi] ; \mathbb{I}_{\left(t_{i}, t_{i+1}\right]}\right\rangle_{\mathcal{L}^{2}(\tilde{\lambda})}\right]=C_{1}+C_{2}, \\
& C_{1}=\mathbb{E}\left[\phi \int_{0}^{T} \sum_{i=1}^{n} \nabla_{s}^{\widetilde{N}^{K}}\left[u_{i}\right] \mathbb{I}_{\left(t_{i}, t_{i+1}\right]}(s) \tilde{\lambda}(\mathrm{d} s)\right]=\mathbb{E}\left[\phi \int_{0}^{T} \sum_{i=1}^{n} \nabla_{s}^{\widetilde{N}^{K}}\left[u_{i} \mathbb{I}_{\left(t_{i}, t_{i+1}\right]}(s)\right] \tilde{\lambda}(\mathrm{d} s)\right] \\
& =\mathbb{E}\left[\phi \int_{0}^{T} \nabla_{s}^{\widetilde{N}^{K}} u_{s} \tilde{\lambda}(\mathrm{d} s)\right] \\
& C_{2}=\mathbb{E}\left[\left\langle\nabla^{\widetilde{N}^{K}}[\phi] ; \sum_{i=1}^{n} u_{i} \mathbb{I}_{\left(t_{i}, t_{i+1}\right]}\right\rangle_{\mathcal{L}^{2}(\tilde{\lambda})}\right]=\mathbb{E}\left[\phi \delta^{\widetilde{N}^{K}}\left(\sum_{i=1}^{n} u_{i} \mathbb{I}_{\left(t_{i}, t_{i+1}\right]}\right)\right]=\mathbb{E}\left[\phi \delta^{\widetilde{N}^{K}}(u)\right] .
\end{aligned}
$$

We then get the following relation:

$$
\mathbb{E}\left[\phi\left(u^{(S L)} \widetilde{N}^{K}\right)_{T}\right]=\mathbb{E}\left[\phi \int_{0}^{T} \nabla_{s}^{\widetilde{N}^{K}} u_{s} \tilde{\lambda}(\mathrm{d} s)\right]+\mathbb{E}\left[\phi \delta^{\tilde{N}^{K}}(u)\right] .
$$

Since this relation holds for all $\phi \in \mathcal{S}$, the result is proved for processes of the form $u=\sum_{i=1}^{n} u_{i} \mathbb{I}_{\left(t_{i}, t_{i+1}\right]}$. The general result follows by a limit procedure when both terms converge.

Remark 2. When $u$ is not of the simple form $u=\sum_{i=1}^{n} u_{i} \mathbb{I}_{\left(t_{i}, t_{i+1}\right]}$, there is no simple condition ensuring the existence of the trace term, i.e., the rightmost integral in (31).

Theorem 20. Let $t \in[0, T]$ be fixed. Consider $\mathcal{K}_{t}^{*}$ the adjoint of $\mathcal{K}$ in $\mathcal{L}_{t}^{2}(\tilde{\lambda}) \stackrel{\text { not }}{=} \mathcal{L}^{2}([0, t], \tilde{\lambda})$.

For any $u \in \mathcal{L}_{t}^{2}(\tilde{\lambda})$, we have:

$$
\mathcal{K}_{t}^{*}(u)=\mathcal{K}_{T}^{*}\left(u \mathbb{I}_{[0, t]}\right) \mathbb{I}_{[0, t]} .
$$


Proof. It is enough to show that for any $g \in \mathcal{L}_{t}^{2}(\tilde{\lambda})$ we have:

$$
\left\langle\mathcal{K}_{t}^{*}(u), g\right\rangle_{\mathcal{L}_{t}^{2}}=\left\langle\mathcal{K}_{T}^{*}\left(u \mathbb{I}_{[0, t]}\right) \mathbb{I}_{[0, t]}, g\right\rangle_{\mathcal{L}_{t}^{2}}
$$

We have,

$$
\begin{aligned}
\left\langle\mathcal{K}_{t}^{*}(u), g\right\rangle_{\mathcal{L}_{t}^{2}(\tilde{\lambda})} & =\langle u, \mathcal{K}(g)\rangle_{\mathcal{L}_{t}^{2}(\tilde{\lambda})}=\left\langle u \mathbb{I}_{[0, t]},\left.\mathcal{K}(g)\right|_{\mathcal{L}^{2}(\tilde{\lambda})}=\left\langle\left[I_{T-}\right]^{-1}\left(u \mathbb{I}_{[0, t]}\right),\left.K(g)\right|_{\mathcal{L}^{2}(\tilde{\lambda})}\right.\right. \\
& =\int_{0}^{T} \int_{0}^{T} K(\tau, v) g(v) \tilde{\lambda}(v) \mathrm{d} v \cdot\left[I_{T-]^{-1}}\left(u \mathbb{I}_{[0, t]}\right)(\tau) \mathrm{d} \tau .\right.
\end{aligned}
$$

We then get:

$$
\left\langle\mathcal{K}_{t}^{*}(u), g\right\rangle_{\mathcal{L}_{t}^{2}(\tilde{\lambda})}=\int_{0}^{T} \int_{0}^{T} K(\tau, v) \cdot\left[I_{T-}\right]^{-1}\left(u \mathbb{I}_{[0, t]}\right)(\tau) \mathrm{d} \tau g(v) \tilde{\lambda}(v) \mathrm{d} v .
$$

Set

$$
\Psi(t, v)=\int_{0}^{T} K(\tau, v) \cdot\left[I_{T-}\right]^{-1}\left(u \mathbb{I}_{[0, t]}\right)(\tau) \mathrm{d} \tau
$$

Let us show that $\Psi(t, v)=0$ as soon as $v \geqslant t$ :

$$
\Psi(t, v)=\int_{v}^{T} K(\tau, v) \cdot\left[I_{T-}\right]^{-1}\left(u \mathbb{I}_{[0, t]}\right)(\tau) \mathrm{d} \tau+\int_{0}^{v} K(\tau, v) \cdot\left[I_{T-}\right]^{-1}\left(u \mathbb{I}_{[0, t]}\right)(\tau) \mathrm{d} \tau .
$$

On the one hand, by triangularity, $K(\tau, v)=0$ as soon as $v \geqslant \tau$ thus the last term is null. On the other hand, $\left[I_{T-}\right]^{-1}\left(u \mathbb{I}_{[0, t]}\right)$ has its support included in $[0, t] \subset[0, v]$. It follows that the first summand is also equal to zero. This implies the following relations:

$$
\Psi(t, v)=K^{*} \circ\left[I_{-T}\right]^{-1}\left(u \mathbb{I}_{[0, t]}\right)(v)=\mathcal{K}^{*}\left(u \mathbb{I}_{[0, t]}\right)(v) \mathbb{I}_{[0, t]}(v) .
$$

The proof is thus complete.

Remark 3. We then have

$$
\left.\delta\left(\mathcal{K}_{t}^{*}(u)\right)-\delta\left(\mathcal{K}_{s}^{*}(u)\right)=\delta\left(\mathcal{K}^{*}\left(u \mathbb{I}_{]} s, t\right]\right)\right)
$$

Hypothesis 2. There exists $\alpha>0$ such that $K$ is continuous, triangular and one-to-one and onto from $\mathcal{L}^{2}$ into the Besov space $\mathcal{I}_{\alpha+1 / 2,2}$ (see Appendix A below).

Hypothesis 3. We assume that the operator $\Gamma$ is continuous from $\mathbb{L}^{2}(\Omega \times[0, T], \mathrm{d} \mathbb{P} \otimes \tilde{\lambda})$ to $\mathbb{L}^{2}(\Omega \times[0, T], \mathrm{d} \mathbb{P} \otimes \tilde{\lambda})$.

Consider the following norm: for any $\Phi \in \mathcal{S}\left(\mathcal{H}^{\tilde{\lambda}}\right)$,

$$
\|\Phi\|_{2,1}^{M}=\mathbb{E}\left[\|\Phi\|_{\mathcal{L}^{2}(\tilde{\lambda})}^{2}\right]+\mathbb{E}\left[\left\|\nabla^{\widetilde{N}} \Phi\right\|_{\mathcal{L}^{2}(\tilde{\lambda}) \otimes \mathcal{L}^{2}(\tilde{\lambda})}^{2}\right]
$$

and define $\mathbb{D}_{2,1}^{M}\left(\mathcal{H}^{\tilde{\lambda}}\right)$ as the closure of $\mathcal{S}\left(\mathcal{H}^{\tilde{\lambda}}\right)$ for this norm.

Proposition 13. Under Assumption 2, we have:

$$
\mathbb{D}_{2,1}^{M}\left(\mathcal{H}^{\tilde{\lambda}}\right) \subset \mathbb{D}_{2,1}^{\tilde{N}}\left(\mathcal{H}^{\tilde{\lambda}}\right) .
$$


Proof. The operator $\Gamma$ is linear and continuous thus

$$
\mathbb{E}\left[\|\Gamma \Phi\|_{\mathcal{L}^{2}(\tilde{\lambda})}^{2}\right] \leqslant c \mathbb{E}\left[\|\Phi\|_{\mathcal{L}^{2}(\tilde{\lambda})}^{2}\right] .
$$

Then, we have

$$
\|\Phi\|_{2,1}^{\widetilde{N}} \leqslant c\left(\mathbb{E}\left[\|\Phi\|_{\mathcal{L}^{2}(\tilde{\lambda})}^{2}\right]+\mathbb{E}\left[\left\|\nabla^{\widetilde{N}} \Phi\right\|_{\mathcal{L}^{2}(\tilde{\lambda}) \otimes \mathcal{L}^{2}(\tilde{\lambda})}^{2}\right]\right),
$$

the result follows.

Theorem 21. For any $\alpha \in\left[1 / 2,1\left[\right.\right.$, under Assumptions 2 and 3, if $u$ belongs to $\mathbb{D}_{p, 1}^{\widetilde{N}}\left(\mathcal{H}^{\tilde{\lambda}}\right) \cap \operatorname{dom}\left(\delta^{K}\right)$ with $\alpha p>1$, then, the process

$$
\left\{\delta^{K}\left(\mathcal{K}_{t}^{*}(u)\right)=\delta^{\widetilde{N}^{K}}\left(u \mathbb{I}_{[0, t]}\right): t \in[0, T]\right\}
$$

admits a modification whose paths are a.s. $(\alpha-1 / p)$-Hölder continuous. Moreover, there exists a constant c $>0$ such that:

$$
\left\|\delta^{K}(u)\right\|_{\mathbb{L}^{2}(\Omega ; \operatorname{Hol}(\alpha-1 / p))} \leqslant c\left\|\mathcal{K}_{T}^{*}\right\|\|u\|_{p, 1}^{\widetilde{N}} .
$$

Proof. Let $(s, t) \in[0, T]^{2}$. By the relation (3) and by continuity of the divergence from $\mathbb{D}_{2,1}^{\widetilde{N}}\left(\mathcal{H}^{\tilde{\lambda}}\right)$ to $\mathbb{L}^{2}(\Omega)$, there exists a constant $c$ such that:

$$
\mathbb{E}\left[\left|\delta^{\widetilde{N}}\left(\mathcal{K}^{*}\left(u \mathbb{I}_{[s, t]}\right)\right)\right|^{2}\right] \leqslant c\left[\left\|\mathcal{K}^{*}\left(u \mathbb{I}_{[s, t]}\right)\right\|_{2,1}^{\widetilde{N}}\right]^{2} \leqslant c\left[\left\|\mathcal{K}^{*}\left(u \mathbb{I}_{[s, t]}\right)\right\|_{2,1}^{M}\right]^{2},
$$

where the last inequality comes from Assumption 2. On the other hand, Assumption 2 of continuity of $K$ from $\mathcal{L}^{2}$ to $\mathcal{I}_{\alpha+1 / 2,2}$ coupled with the fact that $\alpha>\frac{1}{2}$ insure that $\mathcal{K}$ is continuous from $\mathcal{L}^{2}$ into $\mathcal{I}_{\alpha-1 / 2,2}$. Consequently $\mathcal{K}^{*}$ is continuous from $\left[\mathcal{I}_{\alpha-1 / 2,2}\right]^{*}=\mathcal{I}_{1 / 2-\alpha, 2}$ to $\mathcal{L}^{2}$. Denote by $\left\|\mathcal{K}^{*}\right\|$ its operator norm. We have:

$$
\mathbb{E}\left[\left|\delta^{\widetilde{N}}\left(\mathcal{K}^{*}\left(u \mathbb{I}_{[s, t]}\right)\right)\right|^{2}\right] \leqslant c_{1} M\left\|\mathcal{K}^{*}\right\|\left(\mathbb{E}\left[\left\|u \mathbb{I}_{[s, t]}\right\|_{\mathcal{I}_{1 / 2-\alpha, 2}}^{2}\right]+\mathbb{E}\left[\int_{0}^{T}\left\|\nabla^{\widetilde{N}} u \mathbb{I}_{[s, t]}\right\|_{\mathcal{I}_{1 / 2-\alpha, 2}}^{2} \mathrm{~d} r\right]\right) .
$$

According to Proposition 15 with $\beta=\alpha-1 / 2$ and $q=2$, we know that $\mathcal{I}_{\alpha-1 / 2,2}$ is continuously embedded in $\mathcal{L}^{(1-\alpha)^{-1}}$, hence:

$$
\mathcal{L}^{1 / \alpha}=\left[\mathcal{L}^{1 /(1-\alpha)}\right]^{*} \subset\left[\mathcal{I}_{\alpha-1 / 2,2}\right]^{*}=\mathcal{I}_{1 / 2-\alpha, 2} .
$$

Since the embedding is continuous,

$$
\left\|u \mathbb{I}_{[s, t]}\right\|_{\mathcal{I}_{1 / 2-\alpha, 2}} \leqslant c_{2}\left\|u \mathbb{I}_{[s, t]}\right\|_{\mathcal{L}^{1 / \alpha}}
$$

Apply now Hölder inequality to write:

$$
\left\|u \mathbb{I}_{[s, t]}\right\|_{\mathcal{I}_{1 / 2-\alpha, 2}} \leqslant c_{2}\|u\|_{\mathcal{L}^{p}}\left\|\mathbb{I}_{[s, t]}\right\|_{\mathcal{L}^{q}}
$$

with $\frac{1}{\alpha}=\frac{1}{p}+\frac{1}{q}$ (by assumption $\alpha p>1$ ). It is easily seen that $\left\|\mathbb{I}_{[s, t]}\right\|_{\mathcal{L}^{q}}=|t-s|^{\alpha-1 / p}$ hence:

$$
\mathbb{E}\left[\left|\delta^{\tilde{N}}\left(\mathcal{K}^{*}\left(u \mathbb{I}_{[s, t]}\right)\right)\right|^{2}\right] \leqslant c\left\|\mathcal{K}^{*}\right\||t-s|^{\alpha-1 / p} \mathbb{E}\left[\|u\|_{p}+\int_{0}^{T}\left\|\nabla^{\widetilde{N}} u\right\|_{p} \mathrm{~d} r\right] \leqslant c\left\|\mathcal{K}^{*}\right\||t-s|^{\alpha-1 / p}\|u\|_{p, 1}^{\widetilde{N}}
$$

and thus, by the very definition of the norm on Hölder space, we have:

$$
\left\|\delta^{K}(u)\right\|_{\mathbb{L}^{2}(\Omega ; \operatorname{Hol}(\alpha-1 / p))} \leqslant c\left\|\mathcal{K}_{T}^{*}\right\|\|u\|_{p, 1}^{\widetilde{N}},
$$

and the result follows. 


\subsection{Without marks}

In this case, we can proceed as in definition (20):

$$
\hat{\nabla}_{u F}^{K}(v G):=F \mathcal{K}^{*}(v) D G\left(\mathcal{K}^{*}(u)\right)+F G \tilde{\nabla}_{\mathcal{K}^{*}(u)}\left(\mathcal{K}^{*}(v)\right) .
$$

An obvious consequence of relation (19) and Proposition 9 is:

Proposition 14. Let $\Phi \in \mathcal{S}$ and $(\zeta, \phi) \in\left[\mathcal{S}\left(\mathcal{H}^{\lambda *}\right)\right]^{2}$. We have:

$$
D^{\widetilde{N}^{K}}\left(D^{\widetilde{N}^{K}} \Phi(\zeta)\right)(\phi)-D^{\widetilde{N}^{K}}\left(D^{\widetilde{N}^{K}} \Phi(\phi)\right)(\zeta)=D^{\widetilde{N}^{K}} \Phi\left(\hat{\nabla}_{\phi}^{K}(\zeta)-\hat{\nabla}_{\zeta}^{K}(\phi)\right) .
$$

The analog of Theorem 10 cannot be written in terms of covariant derivative but we can state:

Theorem 22. Let $\zeta \in \mathcal{S}\left(\mathcal{H}^{\lambda *}\right)$, we have:

$$
\begin{aligned}
\mathbb{E}\left[\delta^{\widetilde{N}^{K}}(\zeta)^{2}\right] & =\mathbb{E}\left[\left\|\mathcal{K}^{*}(\zeta)\right\|_{\mathcal{L}^{2}(\lambda)}^{2}\right]+\mathbb{E}\left[\operatorname{trace}\left(\hat{\nabla}\left(\mathcal{K}^{*}(\zeta)\right) \circ \hat{\nabla}\left(\mathcal{K}^{*}(\zeta)\right)\right)\right] \\
& =\mathbb{E}\left[\|\zeta\|_{\mathcal{I}^{K}}^{2}\right]+\mathbb{E}\left[\operatorname{trace}\left(\hat{\nabla}\left(\mathcal{K}^{*}(\zeta)\right) \circ \hat{\nabla}\left(\mathcal{K}^{*}(\zeta)\right)\right)\right]
\end{aligned}
$$

To write Weitzenböck formula, it is enough to define an exterior derivative:

$$
d^{K} \phi=d\left(\mathcal{K}^{*}(\phi)\right) \text {. }
$$

The result then follows from Theorem 11:

Theorem 23. Let $\zeta \in \mathcal{S}\left(\mathcal{H}^{\lambda *}\right)$, we have:

$$
\mathbb{E}\left[\delta^{\tilde{N}^{K}}(\zeta)^{2}\right]+\mathbb{E}\left[\left\|d^{K} \zeta\right\|_{\mathcal{L}^{2}(\lambda) \wedge \mathcal{L}^{2}(\lambda)}^{2}\right]=\mathbb{E}\left[\|\zeta\|_{\mathbb{I}^{K}}^{2}\right]+\mathbb{E}\left[\left\|\hat{\nabla}\left(\mathcal{K}^{*} \zeta\right)\right\|_{\mathcal{L}^{2}(\lambda)}^{2}\right]
$$

We can then extend $\mathcal{S}\left(\mathcal{H}^{\lambda *}\right)$ to a larger space named $\mathbb{D}_{2,1}^{C, K}\left(\mathcal{H}^{\lambda *}\right)$ by considering the norm:

$$
\left[\|\zeta\|_{2,1}^{C, K}\right]^{2}=\mathbb{E}\left[\left\|\mathcal{K}^{*}(\zeta)\right\|_{\mathcal{L}^{2}(\lambda)}^{2}\right]+\mathbb{E}\left[\left\|\hat{\nabla}\left(\mathcal{K}^{*}(\zeta)\right)\right\|_{\mathcal{L}^{2}(\lambda)}^{2}\right]
$$

By using this norm, we can avoid Hypothesis 3 of continuity of $\Gamma^{K}$ to establish Theorem 21. In fact, relation (32) is replaced by:

$$
\mathbb{E}\left[\left|\delta^{\widetilde{N}}\left(\mathcal{K}^{*}\left(u \mathbb{I}_{[s, t]}\right)\right)\right|^{2}\right] \leqslant\left[\left\|\mathcal{K}^{*}\left(u \mathbb{I}_{[s, t]}\right)\right\|_{2,1}^{C}\right]^{2},
$$

the rest of the proof remains the same. We get:

Theorem 24. For any $\alpha \in\left[1 / 2,1\left[\right.\right.$, under Assumption 2, if $u$ belongs to $\mathbb{D}_{p, 1}^{C}\left(\mathcal{H}^{\lambda *}\right)$ and to $\operatorname{dom}\left(\delta^{K}\right)$ with $\alpha p>1$. Then, the process

$$
\left\{\delta^{K}\left(\mathcal{K}_{t}^{*}(u)\right)=\delta^{\widetilde{N}^{K}}\left(u \mathbb{I}_{[0, t]}\right): t \in[0, T]\right\}
$$

admits a modification whose paths are a.s. $(\alpha-1 / p)$-Hölder continuous. Moreover, there exists a constant c $>0$ such that:

$$
\left\|\delta^{K}(u)\right\|_{\mathbb{L}^{2}(\Omega ; \operatorname{Hol}(\alpha-1 / p))} \leqslant c\left\|\mathcal{K}_{T}^{*}\right\|\|u\|_{p, 1}^{C} .
$$

\section{Itô formula for cylindrical functionals}

Theorem 25. Let $F$ be a $\mathcal{C}_{b}^{2}$ function and $u$ belong to $\mathcal{S}\left(\mathcal{L}^{2}(v)\right)$, i.e., $u=F . v$ with $F \in \mathcal{S}$ and $v \in \mathcal{L}^{2}(v)$. Consider $Z_{t}=z+\left(u^{(S L)} \tilde{N}^{K}\right)_{t}$. Then $u . F^{\prime} \circ Z$ is in Dom $\delta_{\widetilde{N}^{K}}$ and we have $\mathbb{P}$-almost surely: for any $t \in[0, T]$,

$$
F\left(Z_{t}\right)=F(z)+\left(u(s) F^{\prime}\left(Z_{s}\right)^{(S L)} \tilde{N}^{K}\right)_{t} .
$$


Proof. Consider $\epsilon>0$, we have:

$$
F\left(Z_{t+\epsilon}\right)-F\left(Z_{t}\right)=F^{\prime}\left(Z_{t}\right)\left(Z_{t+\epsilon}-Z_{t}\right)+\left(Z_{t+\epsilon}-Z_{t}\right)^{2} \int_{0}^{T} F^{\prime \prime}\left((1-u) Z_{t+\epsilon}+u Z_{t}\right)(1-u) \mathrm{d} u .
$$

For any test function $\Psi$ we get:

$$
\begin{aligned}
\mathbb{E} & {\left[\left(F\left(Z_{t+\epsilon}\right)-F\left(Z_{t}\right)\right) \cdot \Psi\right] } \\
& =\mathbb{E}\left[F^{\prime}\left(Z_{t}\right)\left(Z_{t+\epsilon}-Z_{t}\right) \cdot \Psi\right]+\mathbb{E}\left[\left(Z_{t+\epsilon}-Z_{t}\right)^{2} \int_{0}^{T} F^{\prime \prime}\left((1-u) Z_{t+\epsilon}+u Z_{t}\right)(1-u) \mathrm{d} u \cdot \Psi\right]=A_{1}+A_{2} .
\end{aligned}
$$

Apply Theorem 19, we get:

$$
\begin{aligned}
Z_{t+\epsilon}-Z_{t} & =\left(u \mathbb{I}_{[t, t+\epsilon]}{ }^{*}{ }^{(S L)} \widetilde{N}^{K}\right)_{T}=\delta^{\widetilde{N}^{K}}\left(u \mathbb{I}_{[t, t+\epsilon]}\right)+\int_{0}^{T} \nabla_{s}^{\widetilde{N}^{K}}\left(u \mathbb{I}_{[t, t+\epsilon]}\right)(s) \tilde{\lambda}(s) \mathrm{d} s \\
& =\delta^{\widetilde{N}}\left(\mathcal{K}^{*}\left(u \mathbb{I}_{[t, t+\epsilon]}\right)\right)+\int_{t}^{t+\epsilon}\left(\mathcal{K} \nabla^{\widetilde{N}}\right)_{s} u(s) \tilde{\lambda}(s) \mathrm{d} s .
\end{aligned}
$$

Thus $A_{1}$ splits into two terms:

$$
A_{1}=\mathbb{E}\left[F^{\prime}\left(Z_{t}\right) \delta^{\widetilde{N}}\left(\mathcal{K}^{*}\left(u \mathbb{I}_{[t, t+\epsilon]}\right)\right) \cdot \Psi\right]+\mathbb{E}\left[F^{\prime}\left(Z_{t}\right) \int_{t}^{t+\epsilon}\left(\mathcal{K} \nabla^{\widetilde{N}}\right)_{s} u(s) \tilde{\lambda}(s) \mathrm{d} s \cdot \Psi\right]=D_{1}+D_{2} .
$$

On the one hand, it is obvious that:

$$
\lim _{\epsilon \rightarrow 0} \frac{1}{\epsilon} D_{2}=\mathbb{E}\left[F^{\prime}\left(Z_{t}\right)\left(\mathcal{K} \nabla^{\tilde{N}}\right)_{t} u(t) \tilde{\lambda}(t) \cdot \Psi\right] .
$$

On the other hand, we have:

$$
D_{1}=\mathbb{E}\left[\int_{0}^{T} \nabla_{s}^{\tilde{N}}\left(F^{\prime}\left(Z_{t}\right) \Psi\right) \mathcal{K}^{*}\left(u \mathbb{I}_{[t, t+\epsilon]}\right) \tilde{\lambda}(s) \mathrm{d} s\right]
$$

An application of the derivation formula splits $D_{1}$ in two terms and using the adjunction property we get:

$$
\begin{aligned}
D_{1} & =\mathbb{E}\left[\int_{0}^{T} \Psi \nabla_{s}^{\widetilde{N}}\left(F^{\prime}\left(Z_{t}\right)\right) \mathcal{K}^{*}\left(u \mathbb{I}_{[t, t+\epsilon]}\right)(s) \tilde{\lambda}(s) \mathrm{d} s\right]+\mathbb{E}\left[\int_{0}^{T} F^{\prime}\left(Z_{t}\right) \nabla_{s}^{\widetilde{N}}(\Psi) \mathcal{K}^{*}\left(u \mathbb{I}_{[t, t+\epsilon]}\right)(s) \tilde{\lambda}(s) \mathrm{d} s\right] \\
& =\mathbb{E}\left[\int_{0}^{T} \Psi\left(\mathcal{K} \nabla^{\widetilde{N}}\right)_{s}\left(F^{\prime}\left(Z_{t}\right)\right) u(s) \mathbb{I}_{[t, t+\epsilon]}(s) \tilde{\lambda}(s) \mathrm{d} s\right]+\mathbb{E}\left[\int_{0}^{T} F^{\prime}\left(Z_{t}\right)\left(\mathcal{K} \nabla^{\tilde{N}}\right)_{s}(\Psi) u(s) \mathbb{I}_{[t, t+\epsilon]}(s) \tilde{\lambda}(s) \mathrm{d} s\right] \\
& =\mathbb{E}\left[\Psi \int_{t}^{t+\epsilon}\left(\mathcal{K} \nabla^{\widetilde{N}}\right)_{s}\left(F^{\prime}\left(Z_{t}\right)\right) u(s) \tilde{\lambda}(s) \mathrm{d} s\right]+\mathbb{E}\left[F^{\prime}\left(Z_{t}\right) \int_{t}^{t+\epsilon}\left(\mathcal{K} \nabla^{\widetilde{N}}\right)_{s}(\Psi) u(s) \tilde{\lambda}(s) \mathrm{d} s\right] .
\end{aligned}
$$

Thus,

$$
\begin{aligned}
& \lim _{\epsilon \rightarrow 0} \frac{1}{\epsilon} \mathbb{E}\left[\Psi \int_{t}^{t+\epsilon}\left(\mathcal{K} \nabla^{\widetilde{N}}\right)_{s}\left(F^{\prime}\left(Z_{t}\right)\right) u(s) \tilde{\lambda}(s) \mathrm{d} s\right]=\mathbb{E}\left[\Psi\left(\mathcal{K} \nabla^{\widetilde{N}}\right)_{t}\left(F^{\prime}\left(Z_{t}\right)\right) u(t) \tilde{\lambda}(t)\right], \\
& \lim _{\epsilon \rightarrow 0} \frac{1}{\epsilon} \mathbb{E}\left[F^{\prime}\left(Z_{t}\right) \int_{t}^{t+\epsilon}\left(\mathcal{K} \nabla^{\widetilde{N}}\right)_{s}(\Psi) u(s) \tilde{\lambda}(s) \mathrm{d} s\right]=\mathbb{E}\left[F^{\prime}\left(Z_{t}\right)\left(\mathcal{K} \nabla^{\widetilde{N}}\right)_{t}(\Psi) u(t) \tilde{\lambda}(t)\right] .
\end{aligned}
$$


Note that:

$$
\left(\mathcal{K} \nabla^{\widetilde{N}}\right)_{t}\left(F^{\prime}\left(Z_{t}\right)\right) u(t)+F^{\prime}\left(Z_{t}\right)\left(\mathcal{K} \nabla^{\widetilde{N}}\right)_{t} u(t)=\left(\mathcal{K} \nabla^{\widetilde{N}}\right)_{t}\left(F^{\prime}\left(Z_{t}\right) u(t)\right) .
$$

Consequently, by the relations (34), (35) and (36), we have shown that:

$$
\lim _{\epsilon \rightarrow 0} \frac{1}{\epsilon} A_{1}=\mathbb{E}\left[F^{\prime}\left(Z_{t}\right)\left(\mathcal{K} \nabla^{\widetilde{N}}\right)_{t}(\Psi) u(t) \tilde{\lambda}(t)\right]+\mathbb{E}\left[\Psi \cdot\left(\mathcal{K} \nabla^{\widetilde{N}}\right)_{t}\left(F^{\prime}\left(Z_{t}\right) u(t)\right) \tilde{\lambda}(t)\right] .
$$

Moreover, since $F^{\prime \prime}$ is bounded,

$$
\lim _{\epsilon \rightarrow 0} \frac{1}{\epsilon} A_{2}=0 .
$$

Thus, we have:

$$
\lim _{\epsilon \rightarrow 0} \frac{1}{\epsilon} \mathbb{E}\left[\left[F\left(Z_{t+\epsilon}\right)-F\left(Z_{t}\right)\right] \cdot \Psi\right]=\mathbb{E}\left[F^{\prime}\left(Z_{t}\right)\left(\mathcal{K} \nabla^{\widetilde{N}}\right)_{t}(\Psi) u(t) \tilde{\lambda}(t)\right]+\mathbb{E}\left[\Psi \cdot\left(\mathcal{K} \nabla^{\widetilde{N}}\right)_{t}\left(F^{\prime}\left(Z_{t}\right) u(t)\right) \tilde{\lambda}(t)\right] .
$$

Using the fundamental lemma of calculus, $F^{\prime}$ being bounded, we can write:

$$
\begin{aligned}
\mathbb{E}\left[\left(F\left(Z_{t}\right)-F(z)\right) \Psi\right] & =\int_{0}^{t} \mathbb{E}\left[F^{\prime}(Z(s)) \Psi\right] \mathrm{d} s=\lim _{\epsilon \rightarrow 0} \int_{0}^{t} \frac{1}{\epsilon} \mathbb{E}\left[\left[F\left(Z_{t+\epsilon}\right)-F\left(Z_{t}\right)\right] \cdot \Psi\right] \mathrm{d} s \\
& =\mathbb{E}\left[\int_{0}^{t} F^{\prime}\left(Z_{s}\right)\left(\mathcal{K} \nabla^{\widetilde{N}}\right)_{s}(\Psi) u(s) \tilde{\lambda}(s) \mathrm{d} s\right]+\mathbb{E}\left[\Psi \cdot \int_{0}^{t}\left(\mathcal{K} \nabla^{\widetilde{N}}\right)_{s}\left(F^{\prime}\left(Z_{s}\right) u(s)\right) \tilde{\lambda}(s) \mathrm{d} s\right] \\
& =\mathbb{E}\left[\int_{0}^{t} \nabla_{s}^{\widetilde{N}}(\Psi) \mathcal{K}_{t}^{*}\left(F^{\prime}\left(Z_{s}\right) u(s)\right) \tilde{\lambda}(s) \mathrm{d} s\right]+\mathbb{E}\left[\Psi \cdot \int_{0}^{t}\left(\mathcal{K} \nabla^{\widetilde{N}}\right)_{s}\left(F^{\prime} \circ Z(s) \cdot u(s)\right) \tilde{\lambda}(s) \mathrm{d} s\right] \\
& =\mathbb{E}\left[\Psi \cdot \delta^{\widetilde{N}}\left(\mathcal{K}_{t}^{*}\left(F^{\prime}\left(Z_{s}\right) u(s)\right)\right)\right]+\mathbb{E}\left[\Psi \cdot \int_{0}^{t}\left(\mathcal{K} \nabla^{\widetilde{N}}\right)_{s}\left(F^{\prime} \circ Z(s) \cdot u(s)\right) \tilde{\lambda}(s) \mathrm{d} s\right] .
\end{aligned}
$$

We then apply Theorem 20 to write:

$$
\begin{aligned}
\mathbb{E} & {\left[\left(F\left(Z_{t}\right)-F(z)\right) \Psi\right] } \\
& =\mathbb{E}\left[\Psi \cdot \delta^{\tilde{N}}\left(\mathcal{K}^{*}\left(F^{\prime}\left(Z_{s}\right) u(s) \mathbb{I}_{[0, t]}(s)\right)\right)\right]+\mathbb{E}\left[\Psi \cdot \int_{0}^{t}\left(\mathcal{K} \nabla^{\tilde{N}}\right)_{s}\left(F^{\prime} \circ Z(s) \cdot u(s)\right) \tilde{\lambda}(s) \mathrm{d} s\right] \\
& =\mathbb{E}\left[\Psi \cdot \delta^{\tilde{N}^{K}}\left(F^{\prime}\left(Z_{s}\right) u(s) \mathbb{I}_{[0, t]}(s)\right)\right]+\mathbb{E}\left[\Psi \cdot \int_{0}^{t}\left(\mathcal{K} \nabla^{\tilde{N}}\right)_{s}\left(F^{\prime} \circ Z(s) \cdot u(s)\right) \tilde{\lambda}(s) \mathrm{d} s\right] \\
& =\mathbf{E}\left[\Psi \cdot\left(\delta^{T}\left(F^{\prime} \circ Z(s) \cdot u(s) \mathbb{I}_{[0, t]}(s)\right)+\int_{0}^{T}\left(\mathcal{K} \nabla^{\widetilde{N}}\right)_{s}\left(F^{\prime} \circ Z(s) \cdot u(s)\right) \mathbb{I}_{[0, t]}(s) \tilde{\lambda}(s) \mathrm{d} s\right)\right] \\
& =\mathbb{E}\left[\Psi \cdot\left(F^{\prime} \circ Z \cdot u^{*}{ }^{(S L)} \tilde{N}^{K}\right)_{t}\right] .
\end{aligned}
$$

The relation (33) is obtained by identification for all $t \in[0, T] \mathbb{P}$-a.e. but both terms are continuous so it is true $\mathbb{P}$-a.s. for all $t \in[0, T]$. 


\section{Appendix A. Deterministic fractional calculus}

For $f \in \mathcal{L}^{1}([0, T])$, the left and right fractional integrals of $f$ are defined by:

$$
\begin{array}{ll}
\left(I_{0^{+}}^{\alpha} f\right)(x)=\frac{1}{\Gamma(\alpha)} \int_{0}^{x} f(t)(x-t)^{\alpha-1} \mathrm{~d} t, & x \geqslant 0, \\
\left(I_{b^{-}}^{\alpha} f\right)(x)=\frac{1}{\Gamma(\alpha)} \int_{x}^{b} f(t)(t-x)^{\alpha-1} \mathrm{~d} t, & x \leqslant b,
\end{array}
$$

where $\alpha>0$ and $I^{0}=$ Id.

For any $\alpha \geqslant 0$, any $f \in \mathcal{L}^{p}([0, T])$ and $g \in \mathcal{L}^{q}([0, T])$ where $p^{-1}+q^{-1} \leqslant \alpha$, we have:

$$
\int_{0}^{T} f(s)\left(I_{0^{+}}^{\alpha} g\right)(s) \mathrm{d} s=\int_{0}^{T}\left(I_{T^{-}}^{\alpha} f\right)(s) g(s) \mathrm{d} s .
$$

The Besov space $I_{0^{+}}^{\alpha}\left(\mathcal{L}^{p}\right) \stackrel{\text { not }}{=} \mathcal{I}_{\alpha, p}$ is usually equipped with the norm:

$$
\|f\|_{\mathcal{I}_{\alpha, p}}=\left\|I_{0^{+}}^{-\alpha} f\right\|_{\mathcal{L}^{p}} .
$$

In particular $\mathcal{I}_{\alpha, 2}$ is a (separable) Hilbert space and we have the following continuity results (see [9,20]):

\section{Proposition 15.}

- If $\alpha-1 / p<0$, then $\mathcal{I}_{\alpha, p}$ is isomorphic to $I_{T^{-}}^{\alpha}\left(\mathcal{L}^{p}\right)$.

- If $0<\alpha<1,1<p<1 / \alpha$, then $I_{0^{+}}^{\alpha}$ is a bounded operator from $\mathcal{L}^{p}([0, T])$ into $\mathcal{L}^{q}([0, T])$ with $q=$ $p(1-\alpha p)^{-1}$.

- For any $0<\alpha<1$ and any $p \geqslant 1, \mathcal{I}_{\alpha, p}$ is continuously embedded in $\operatorname{Hol}(\alpha-1 / p)$ provided that $\alpha-1 / p>0$. For $0<v \leqslant 1, \operatorname{Hol}(v)$ denotes the space of Hölder-continuous functions, null at time 0 , equipped with the norm:

$$
\|f\|_{\operatorname{Hol}(v)}=\sup _{t \neq s} \frac{|f(t)-f(s)|}{|t-s|^{v}} .
$$

\section{References}

[1] S. Albeverio, Y.G. Kondratiev, M. Rockner, Analysis and geometry on configuration spaces, J. Funct. Anal. 154 (2) (1998) $444-500$.

[2] A. Benassi, S. Cohen, J. Istas, Identification and properties of real harmonizable fractional Lévy motions, Bernoulli 8 (1) (2002) $97-115$.

[3] A. Benth, F.E. Lokka, Anticipative calculus for Lévy processes and stochastic differential equations, Stochastics Stochastics Rep. 76 (3) (2004) 191-211.

[4] G. Chobanov, Modeling financial asset returns with shot noise processes, Math. Comput. Modelling 29 (10-12) (1999) 17-21.

[5] A. Dassios, J. Jang, The shot-noise process and its distribution at claim times as the intensity of a doubly stochastic process, Preprint, 2001.

[6] L. Decreusefond, Perturbation analysis and Malliavin calculus, Ann. Appl. Probab. 8 (2) (1998) 496-523.

[7] L. Decreusefond, A.S. Üstünel, Stochastic analysis of the fractional Brownian motion, Potential Anal. 10 (2) (1999) $177-214$.

[8] G. Di Nunno, B. Øksendal, F. Proske, White noise analysis for Lévy processes, J. Funct. Anal. 206 (1) (2004) $109-148$.

[9] D. Feyel, A. de La Pradelle, On fractional Brownian processes, Potential Anal. 10 (3) (1999) 273-288.

[10] J. Jacod, Calcul stochastique et problèmes de martingales, Lecture Notes in Math., vol. 714, Springer, Berlin, 1979.

[11] G. Köthe, Topological Vector Spaces, A Series of Comprehensive Studies in Mathematics, Springer-Verlag, 1979.

[12] J.A. Léon, J. Solé, F. Utzet, J. Vives, On Lévy processes, Malliavin calculus and market models with jumps, Finance Stochast. 6 (2002) 197-225.

[13] D. Nualart, J. Vives, Anticipative calculus for the Poisson process based on the Fock space, in: Séminaire de Probabilités 1988/89, vol. XXIV, Springer, Berlin, 1990, pp. 154-165.

[14] E. Parzen, Stochastic Processes, Classics Appl. Math., vol. 24, Society for Industrial and Applied Mathematics (SIAM), Philadelphia, PA, 1999 (reprint of the 1962 original).

[15] J.-J. Prat, N. Privault, Explicit stochastic analysis of Brownian motion and point measures on Riemannian manifolds, J. Funct. Anal. 167 (1) (1999) 201-242. 
[16] N. Privault, Chaotic and variational calculus in discrete and continuous time for the Poisson process, Stochastics Stochastics Rep. 51 (1-2) (1994) 83-109.

[17] N. Privault, Connection, parallel transport, curvature and energy identities on spaces of configurations, C. R. Acad. Sci. Paris, Sér. I Math. 330 (10) (2000) 899-904.

[18] N. Privault, Connections and curvature in the Riemannian geometry of configuration spaces, J. Funct. Anal. 185 (2) (2001) $367-403$.

[19] N. Privault, Weitzenböck formulas on Poisson probability spaces, in: Geometry, Integrability and Quantization, Varna, 2001, Coral Press Sci. Publ., Sofia, 2002, pp. 382-394.

[20] S.G. Samko, A. Kilbas, O. Marichev, Fractional Integrals and Derivatives, Gordon and Breach Science Publishers, Yverdon, 1993.

[21] G. Samorodnitsky, A class of shot-noise models for financial applications, in: Athens Conference on Applied Probability and Time Series Analysis, in: Lecture Notes in Statist., vol. I, Springer-Verlag, New York, 1996, pp. 332-353.

[22] D. Snyder, M.I. Miller, Random Point Processes in Time and Space, Springer-Verlag, New York, 1991.

[23] S. Yue, M. Hishino, The general cumulants for a filtered point processes, Appl. Math. Modelling 25 (2001) 193-201. 